Se assumirmos que a indentação é linear com o nível da fibra (ZHANG, 1999), para a região de uma dobra completa, $z \in[0,2 H]$ :

$$
\delta_{z}(z)=\left(\frac{z}{2 H}\right) \delta ; \quad z \in[0,2 H]
$$

obtemos:

$$
\begin{gathered}
\varepsilon_{I I I}=\frac{1}{2}\left(\frac{z}{2 H}\right)^{2}\left(\frac{\delta}{b_{1}}\right)^{2} \mathrm{e} \\
\dot{\varepsilon}_{I I I}=\left(\frac{z}{2 H}\right)^{2} \frac{\delta \dot{\delta}}{b_{1}^{2}}
\end{gathered}
$$

a deformação e taxa de deformação em função da posição $z$ da fibra e de $\delta$ e $\dot{\delta}$. Utilizando o conceito de Fluxo de Energia, Eq. 5.14, e o critério de Von Mises para Tração, Eq. 5.27, no mecanismo em questão:

$$
\dot{E}_{\mathrm{III}}=\iint_{S 1} \frac{2}{\sqrt{3}} \sigma_{0} t \dot{\varepsilon}_{I I I} \mathrm{~d} S
$$

$\operatorname{com} d S=2 H b_{1} t$

$$
\dot{E}_{\mathrm{III}}=\iint_{S 1} \frac{2}{\sqrt{3}} \sigma_{d} t\left(\frac{z}{2 H}\right)^{2}\left(\frac{\delta \dot{\delta}}{b_{1}^{2}}\right) \mathrm{d} S
$$

Resolvendo, obtém-se o fluxo de energia em função do deslocamento $\delta$, e velocidade instantânea $\dot{\delta}$.

$$
\dot{E}_{\mathrm{m}, \mathrm{III}}=\frac{4}{3 \sqrt{3}} \sigma_{d} t H \frac{\delta \dot{\delta}}{b_{1}} .
$$

Ao dividir a Eq. 5.58 por $\dot{\delta}$, obtém-se a expressão da força por deslocamento:

$$
F_{\mathrm{m}, \mathrm{III}}=\frac{4}{3 \sqrt{3}} \sigma_{d} t H \frac{\delta}{b-k H} .
$$




\subsubsection{Implementação Numérica}

A absorção de energia pelo conjunto é a soma da energia das rótulas plásticas lineares; das regiões I, II e III, além da chapa superior. Da mesma forma, a força total é composição das forças dos mecanismos citados. Parte das equações está em função de $\delta$ e $\dot{\delta}$ instantâneos, sendo necessária implementação numérica (Fig. 5.13a) para avaliar as equações ponto a ponto, ao longo do deslocamento.

E necessário fornecer como entrada no programa: as dimensões do painel e o fator de escala, as informações do ensaio como massa e velocidade de impacto e as propriedades do material. Para simplificar o processo iterativo, é assumido que a velocidade decai linearmente, com uma estimativa inicial de deslocamento máximo, conforme gráfico da Fig. 5.13b.

Assim, supondo um deslocamento máximo $\delta_{\max }$, a velocidade inicial $V_{0}$ varia linearmente até atingir 0 , em $\delta_{\max }$. A força é calculada para diversos valores de deslocamento, usando as equações da seção anterior, até $\delta_{\max }$. O resultado de força é, então, integrado para se obter a energia absorvida pelo painel. Caso esta energia absorvida for diferente - dentro de uma tolerância - da energia cinética inicial, o valor de deslocamento máximo é alterado até que convirja. Esse procedimento é realizado tanto para o painel protótipo quanto para os modelos VSG e MLT, visto que o $\delta_{\max }$ varia para cada um deles. O processo de solução necessita de 40 iterações para convergir e leva em torno de 5 minutos.

\subsection{Resultados}

Os dados nominais do ensaio do painel, Capítulo 3, e os parâmetros iniciais característicos da implementação, Tabela 5.1, foram inseridos no programa e forneceram os resultados das Figs. 5.14-5.16, para painel protótipo, MLT e VSG, respectivamente.

Tabela 5.1 Entradas do programa.

\begin{tabular}{llll}
\hline Entradas do Programa & Protótipo & MLT & VSG \\
\hline Velocidade Inicial, $V_{0}[\mathrm{~m} / \mathrm{s}]$ & 7,0 & 7,0 & 5,6 \\
\hline Massa de Impacto, $G[\mathrm{Kg}]$ & 1000 & 0,5787 & 0,699 \\
$\delta_{\text {estimativa inicial }}[\mathrm{mm}]$ & 161 & 13,4 & 13,4 \\
\hline
\end{tabular}

Todas as curvas apresentam características similares. Para o mecanismo 1 - de rótulas plásticas - próximo a deslocamento nulo, i.e., $\alpha=0^{\circ}$, a força tende a infinito, como 


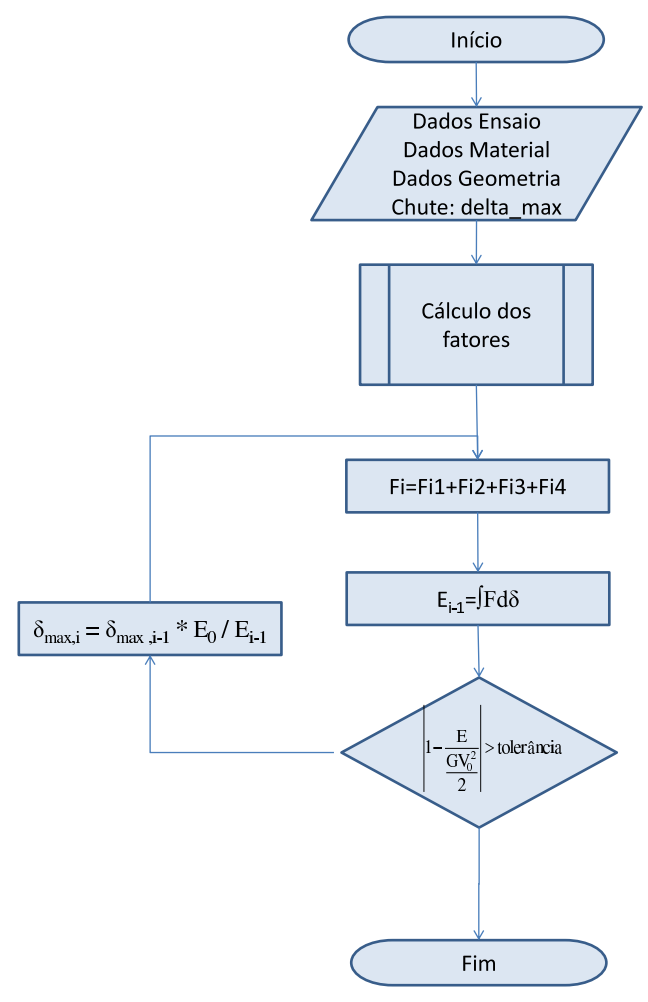

(a) Fluxograma do programa escrito em Matlab de composição da força e energia totais a partir dos mecanismos individuais.

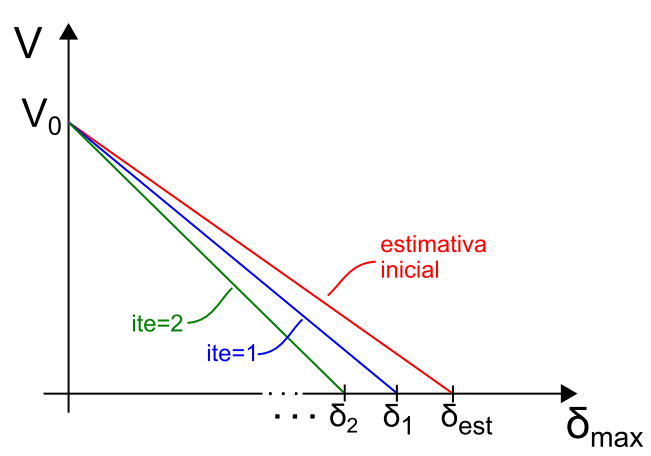

(b) Processo iterativo de estabelecimento do deslocamento máximo e queda da velocidade ao longo do deslocamento.

Figura 5.13 Processo iterativo para estabelecer a curva de força por deslocamento e energia do painel reforçado.

esperado; e decai continuamente até o final do cálculo. O mecanismo 2 - que compreende as regiões I e II, comparado aos demais, não contribui significativamente para a força total; ao passo que o de número 3 - de deformação no plano das chapas - contribui com cerca de $1 / 3$ da força computada ponto-a-ponto. É também para esse mecanismo que surgem as principais diferenças entre os resultados para as leis viscoplásticas de Cowper-Symonds, Figs. 5.14a, 5.15a e 5.16a e as de Norton, Figs. 5.14b, 5.15b e 5.16b. Para os modelos, a inclinação da queda é mais evidente para a lei de Cowper-Symonds; enquanto que, para o protótipo, a diferença principal é no nível da força. Com relação ao último mecanismo, contra esforço de membrana - número 4, cresce conforme o deslocamento do indentador, tendendo a se estabilizar em um valor máximo, devido à velocidade que tende a zero.

Para o MLT usando Cowper-Symonds, Fig. 5.15a, há energia excedente para iniciar uma segunda dobra. Também é possível observar a manutenção dos níveis de força para os mecanismos 3 e 4 e o caráter cíclico do mecanismo 1. Sobre a força total dos modelos de ambas as leis, Figs. 5.15 e 5.16, a tendência de queda dos mecanismos 1 e 3 é contra- 


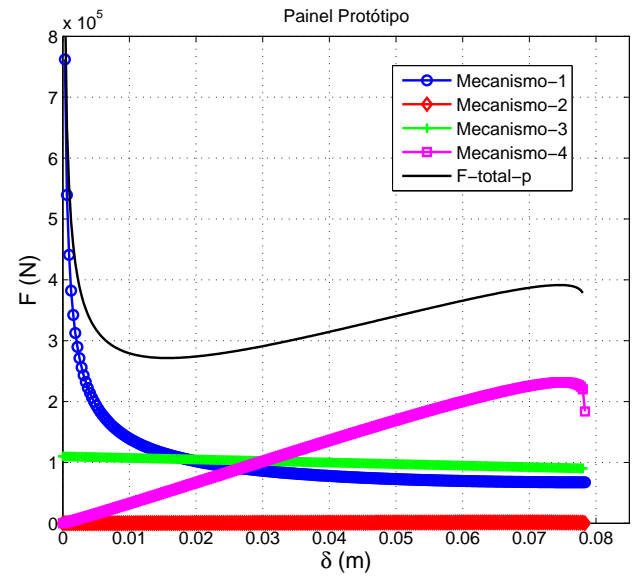

(a) Protótipo - Cowper-Symonds.

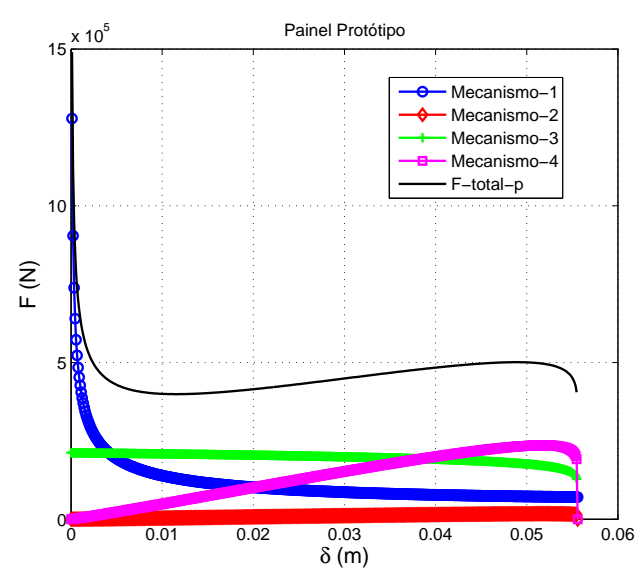

(b) Protótipo - Norton.

Figura 5.14 Resultados do método analítico para o protótipo utilizando duas leis de viscoplasticidade diferentes.

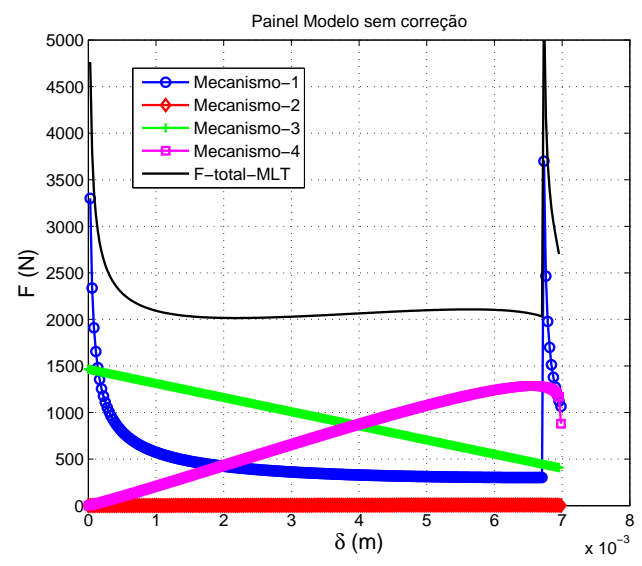

(a) Modelo sem correção - CowperSymonds.

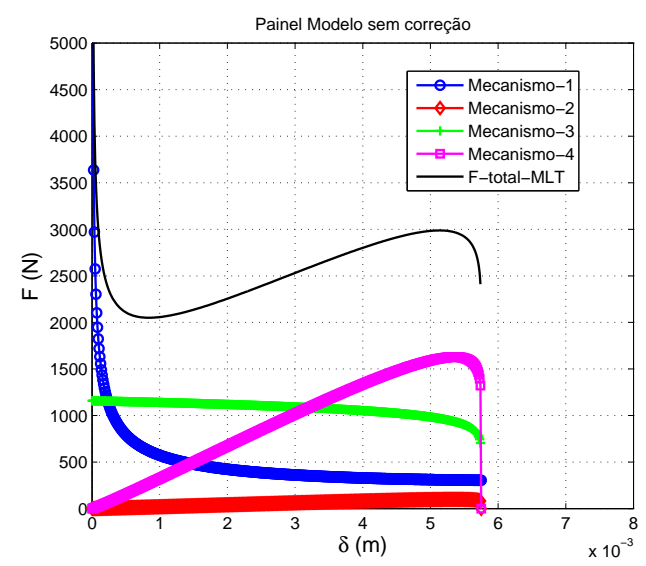

(b) Modelo sem correção - Norton.

Figura 5.15 Resultados do método analítico para o modelo sem correção utilizando duas leis de viscoplasticidade diferentes.

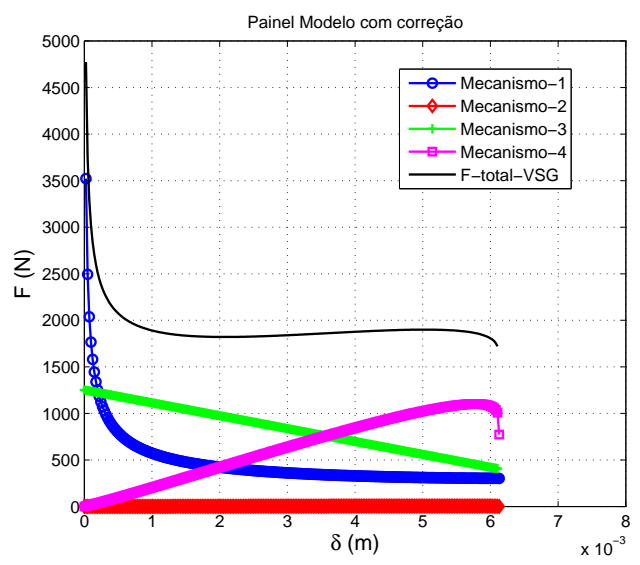

(a) Modelo com correção - CowperSymonds.

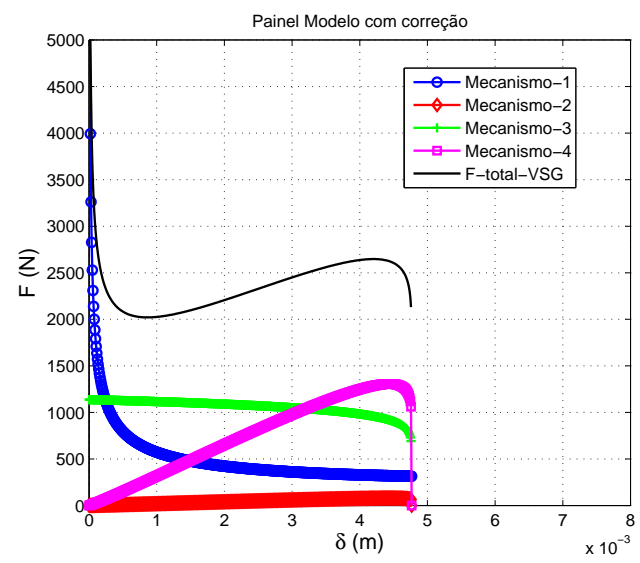

(b) Modelo com correção - Norton.

Figura 5.16 Resultados do método analítico para o modelo corrigido utilizando duas leis de viscoplasticidade diferentes. 
balanceada pelo mecanismo 4. Para os que utilizam a lei de Cowper-Symonds, são quase equivalentes; para os de Norton, o esforço de membrana é maior, aumentando a força total conforme o deslocamento aumenta.

Comparando o aspecto das curvas obtidas por Norton, Figs. 5.14b, 5.15b e 5.16b, é possível observar que possuem forma similar, com a mesma tendência de acréscimo da força; tanto para os modelo como para os protótipos, e mostram maior rigidez se comparados às de Cowper-Symonds. Contudo, as curvas de Cowper-Symonds mostram um comportamento não similar entre protótipo Fig. 5.14a, e modelos, Figs. 5.15a e 5.16a.

Para tornar as curvas analíticas próximas às obtidas nos ensaios foi necessário suavizar o início. Ou seja, por causa do mecanismo 1, a soma tende a infinito para valores de $\delta$ próximo a zero ou $2 H$, tornando os valores de energia irreais. Assim, as curvas obtidas analiticamente tiveram os valores de força alterados para remover essa tendência de infinito para valores de $\delta \rightarrow 0$. Definindo o primeiro ponto $F(\delta=0)=0$ e suavizando sucessivamente, mantendo um pico chegou-se às curvas das Figs 5.17 e 5.18. Esse procedimento tornou possível o cálculo de energia, $E=\int F \mathrm{~d} \delta$, que fora também utilizado para convergência do algoritmo.

Os resultados experimentais do protótipo são comparados aos obtidos analiticamente, Fig. 5.17a e Tabela 5.2. No início da curva, $\delta=[0 ; 0,02]$; o modelo analítico capta razoavelmente bem o comportamento obtido através de ensaio, sendo bastante distinto da porção final. Lembrando as premissas do equacionamento analítico - impacto central vertical e sem fratura -, é compreensível a diferença de resultados, uma vez que eles não representam o mesmo fenômeno. O mesmo pode ser observado para os resultados usando Cowper-Symonds, Fig. 5.18a, apesar do maior deslocamento.

Os resultados experimentais e analíticos (Norton) para o painel MLT, Fig. 5.17b, mostram coerência tanto na forma da curva, como para os níveis de força e deslocamento. Adicionalmente, para maior parte da curva, pode-se dizer que a analítica estabelece o nível máximo de força para o qual a estrutura resiste, i.e., limite superior, como considerado inicialmente no equacionamento. Ademais, nota-se um atraso na subida do nível de força na curva experimental, sinal de que há acomodações do painel, porção elástica e também a atuação do filtro; todos não considerados analiticamente. Similarmente, o painel VSG possui curvas representativas do experimento com boa correlação com a analítica de Norton. É mais facilmente observável um possível efeito da falha parcial da solda da cruciforme, que torna a subida da força experimental entre $\delta=3,8 \sim 4,5$, não tão alta quanto a analítica, Fig. 5.17c, ou como a ocorrida na MLT, Fig. 5.17b, ainda que os deslocamentos máximos tenham sido próximos, Tabela 5.2. 


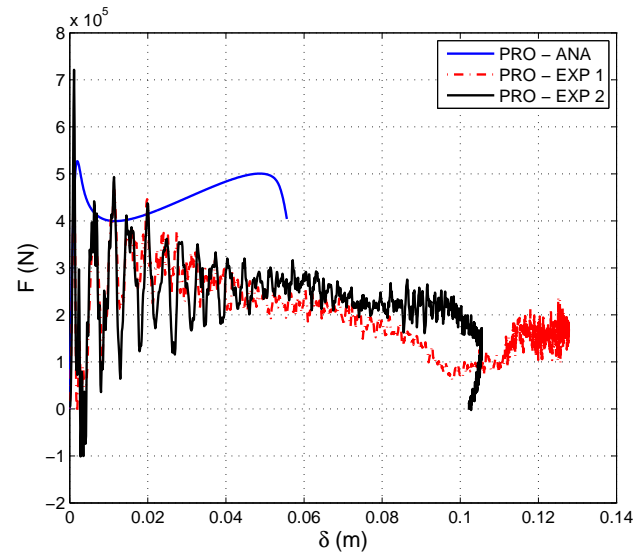

(a) Protótipo

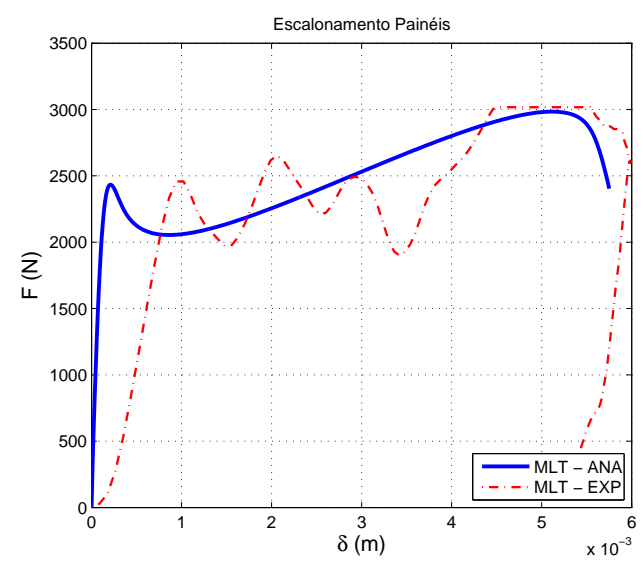

(b) Modelo MLT - sem correção

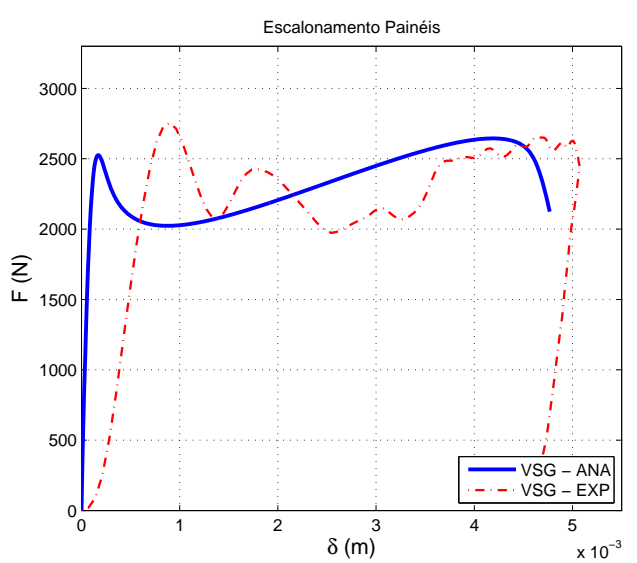

(c) Modelo VSG - Corrigido

Figura 5.17 Comparativo entre experimentos de protótipo e modelos e as curvas obtidas analiticamente utilizando a lei viscoplástica de Norton.

Tabela 5.2 Comparação dos deslocamentos máximos obtidos experimental e analiticamente.

\begin{tabular}{lccccc}
\hline & $\begin{array}{c}\text { Experimental } \\
\delta_{\max }[\mathrm{mm}]\end{array}$ & \multicolumn{2}{c}{ Cowper-Symonds } & \multicolumn{2}{c}{ Norton } \\
& $\delta_{\max }[\mathrm{mm}]$ & $\begin{array}{c}\text { Diferença } \\
{[\%]}\end{array}$ & $\delta_{\max }[\mathrm{mm}]$ & $\begin{array}{c}\text { Diferença } \\
{[\%]}\end{array}$ \\
\hline Protótipo 1 & 127,8 & 85,63 & $-33,0$ & 67,7 & $-47,1$ \\
Protótipo 2 & 105,5 & 85,63 & $-18,8$ & 67,7 & $-35,9$ \\
MLT & 6,0 & 7,8 & 30,2 & 6,6 & 10,2 \\
VSG & 5,7 & 7,0 & 21,8 & 5,7 & 0,5 \\
\hline
\end{tabular}

Para as curvas usando CS, Fig. 5.18b e c, i.e., o valor da força fica a maior parte do deslocamento abaixo dos resultados experimentais. Além disso, há o início de uma segunda dobra em ambas as curvas analíticas; algo que não foi observado como sendo tão desenvolvido como se faz supor tais curvas.

Utilizando as leis de similaridade do Capítulo 2, é possível correlacionar modelos e pro- 


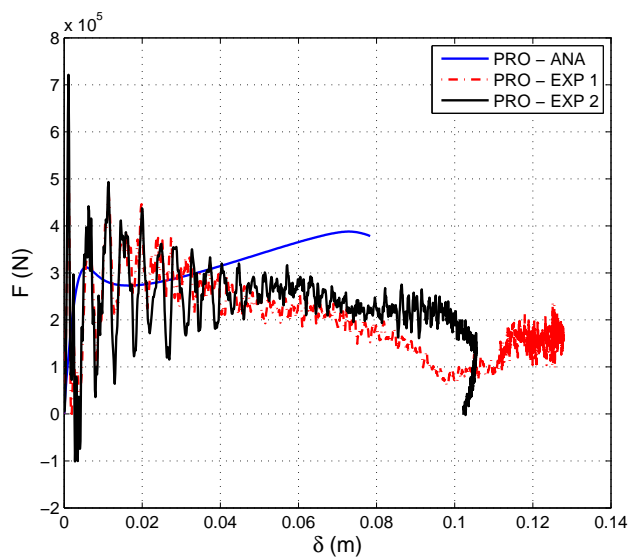

(a) Protótipo.

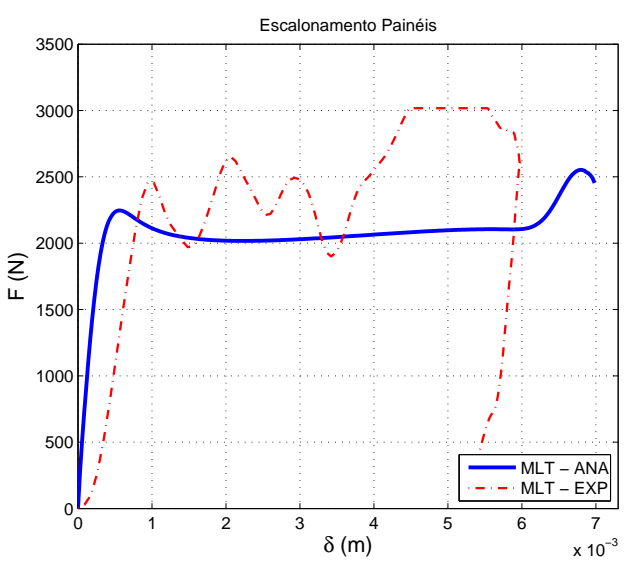

(b) Modelo MLT - sem correção.

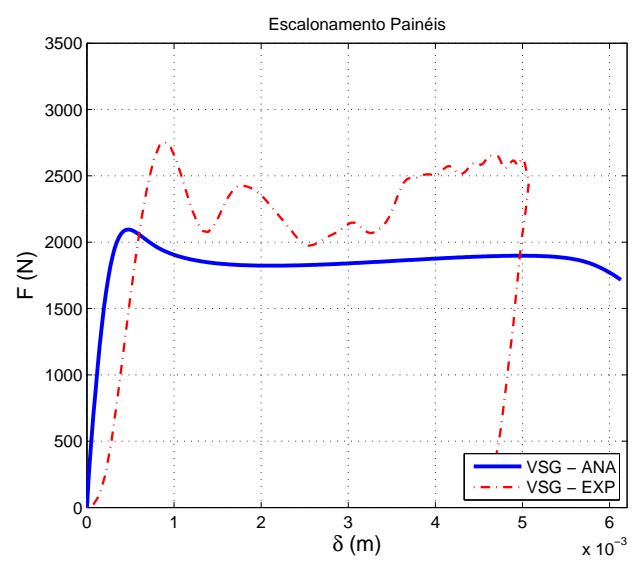

(c) Modelo VSG - corrigido.

Figura 5.18 Comparativo entre experimentos de protótipo e modelos e as curvas obtidas analiticamente utilizando a lei viscoplástica de CowperSymonds.

tótipos. As Figs. 5.19 e 5.20 mostram as curvas de Energia vs. Deslocamento e Força vs. Deslocamento obtidas analiticamente - corrigidas pelos fatores correspondentes - usando lei viscoplásticas de Norton e de Cowper-Symonds, respectivamente. Na Fig. 5.19a notase que a resposta do modelo corrigido é mais próxima da do protótipo que a do modelo sem correção. Além disso, elas se aproximam mais com o crescimento do deslocamento, o que pode ser explicado pela influência do mecanismo 1. A queda de sua influência na força total a partir de $\delta=0,03$ (Fig. 5.14b) colabora para uma melhor correlação entre modelo e protótipo, porquanto as equações consideram a sensibilidade à taxa de deformação, algo não modelado para este mecanismo. Observa-se também boa aproximação do deslocamento máximo e da forma geral da curva.

A reduzida disparidade no início das curvas de Força vs. Deslocamento do protótipo em preto - e VSG - em vermelho, Fig. 5.19a, reflete nas curvas de $E$ vs. $\delta$, Fig. 5.19b. Até $\delta=0,03 \mathrm{~m}$, há tendência de destacamento das curvas, que deixa de crescer e se mantém constante até o deslocamento máximo. Isso mostra que a correlação usando as leis de 
similaridade forneceu resultados do modelo VSG mais próximos do protótipo do que os do MLT quando usada a lei viscoplástica de Norton.

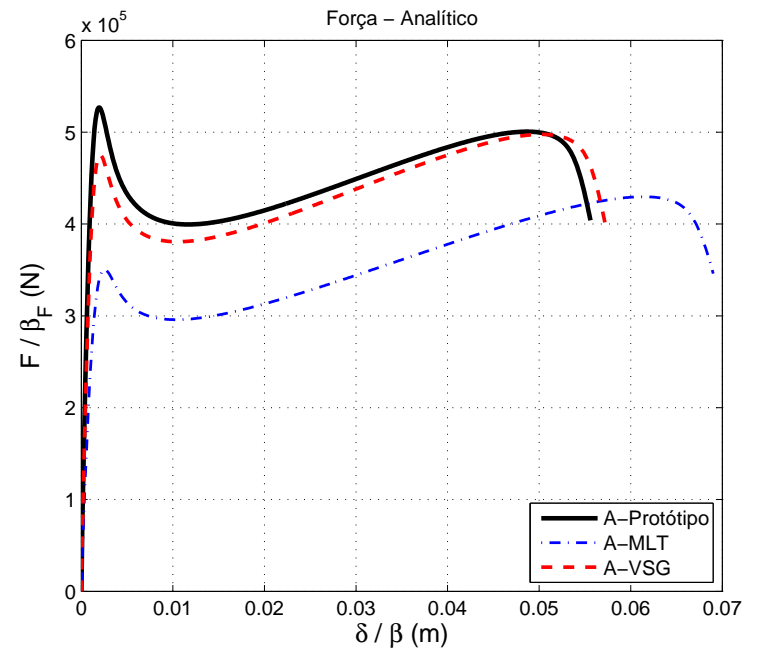

(a) Força vs. Deslocamento.

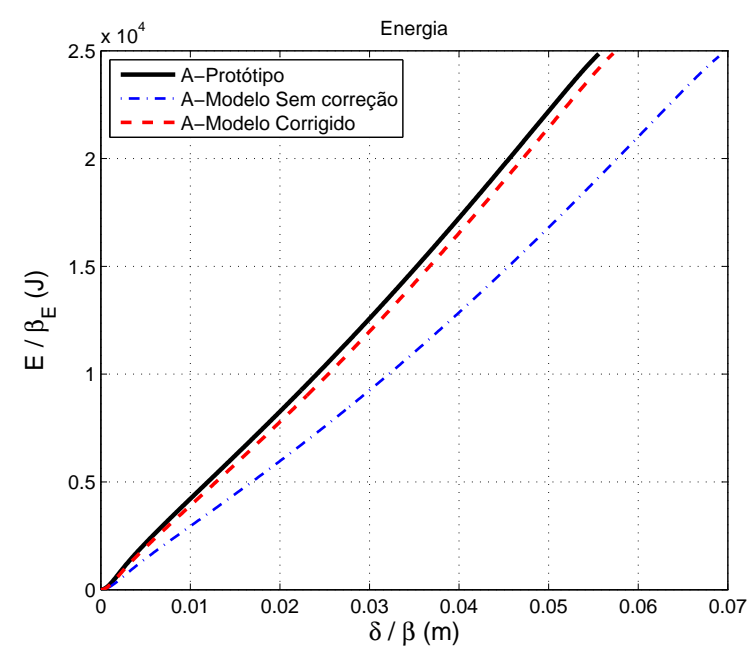

(b) Energia vs. Deslocamento.

Figura 5.19 Correlação analítica entre modelo e protótipo utilizando lei viscoplástica de Norton.

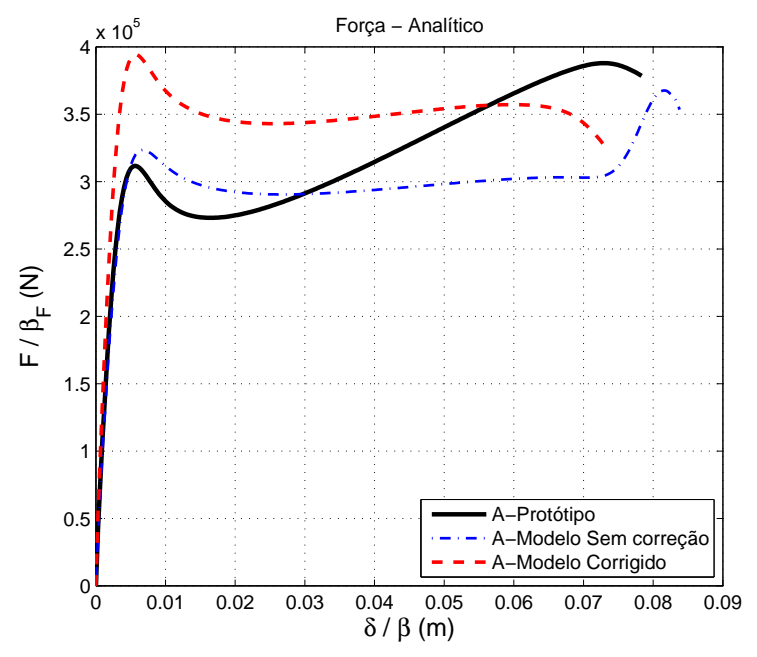

(a) Força vs. Deslocamento.

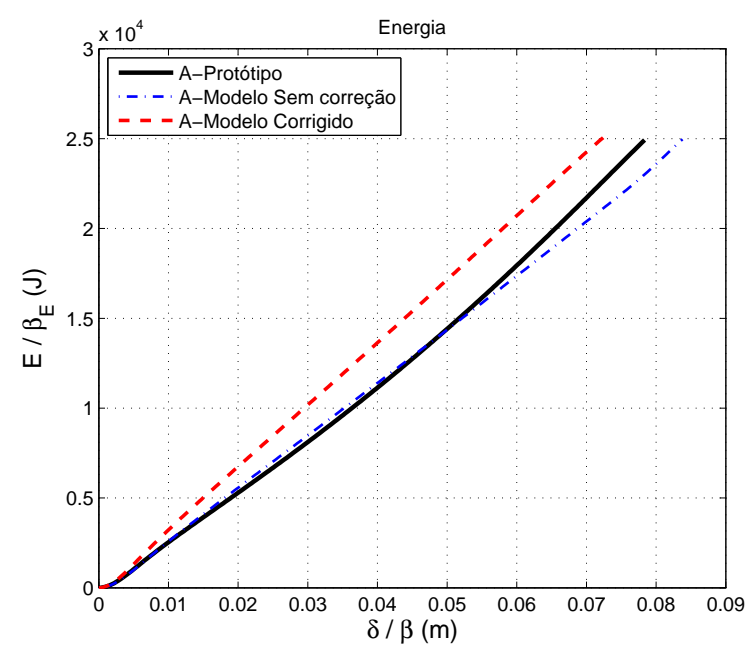

(b) Energia vs. Deslocamento.

Figura 5.20 Correlação analítica entre modelo e protótipo utilizando lei viscoplástica de Cowper-Symonds.

Para as curvas obtidas com a formulação de Cowper-Symonds, Figs. 5.20a e b, há disparidades significantes. As curvas correspondentes aos esforços de cada mecanismo diferem entre modelos e protótipo. Isto reflete em um início de curva inferior para o protótipo, $\delta=0,1$, devido à $\dot{\varepsilon}$, e um crescimento abrupto a partir de $\delta=0,3$. Neste ponto, os mecanismos 3 e 4 se contrabalanceiam nos modelos, ao passo que no protótipo o 3 permanece quase constante e o 4, aumenta; resultando na forma "plana" para os primeiros e crescente para o protótipo.

Isso posto, para um caso analítico utilizando Cowper-Symonds, nesse nível de taxa de 
deformação e para essa diferença de material, a correlação não fornece bons resultados, tanto para o modelo MLT como para o VSG. Quanto às curvas de energia, Fig. 5.20b, o modelo não corrigido se aproxima mais do protótipo ainda que possuam curvaturas e comportamento diferentes. 



\section{Modelagem Numérica}

Neste capítulo são mostradas de forma breve as particularidades do método de integração explícita e suas implicações na criação do modelo numérico. Em seguida, as características do painel reforçado são discutidas, em busca de uma forma de reproduzir os achados experimentais do Capítulo 4. Por fim, os resultados obtidos numericamente são comparados aos experimentais e aos analíticos.

\subsection{Método dos Elementos Finitos}

O equilíbrio dinâmico de um corpo pode ser escrito como sendo o equilíbrio entre as forças internas e externas, ou seja (BATHE, 1996):

$$
\mathbf{M} \ddot{\mathbf{U}}+\mathbf{C} \dot{U}+\mathbf{K U}=\mathbf{R}
$$

onde $\mathbf{M}, \mathbf{C}, \mathbf{K}$ são as matrizes de massa, amortecimento e rigidez, respectivamente, e $\mathbf{R}$ é o vetor de forças externas aplicadas ao sistema. A Eq. 6.1 pode ainda ser escrita como (BATHE, 1996):

$$
\begin{aligned}
\mathbf{M} \ddot{u}(t)+\mathbf{C} \dot{u}(t)+\mathbf{K} u(t) & =R(t) \\
F_{I}(t)+F_{D}(t)+F_{E}(t) & =R(t),
\end{aligned}
$$

sendo $F_{I}(t)$ as forças inerciais, $F_{D}(t)$ as de amortecimento e $F_{E}(t)$, as elásticas; todas 
em função do tempo. Dessa forma, se há o equilíbrio para cada instante de tempo $t$, é necessário integrar no tempo para se obter a resposta para os instantes do intervalo desejado. Existem duas formas de se integrar no tempo a Eq. 6.2a no método de elementos finitos: a explícita e a implícita (WRIGGERS, 2008).

Na implícita, o procedimento substitui as derivadas em função do tempo por "quantidades" que dependem do instante anterior e de "quantidades" não conhecidas no tempo posterior (instantes seguintes, $t_{n+\alpha}$ ). A vantagem desse método reside em ser incondicionalmente estável, sendo sua desvantagem requerer a solução de um sistema de equações não-linear a cada incremento tempo, demandando alto custo computacional.

Na explícita, a solução do instante seguinte $\left(t_{n+1}\right)$ depende somente do tempo atual $\left(t_{n}\right)$, simplificando a implementação. É menos custoso por não necessitar a solução de um sistema não linear, e sim somente a inversão da matriz de massa (ainda mais simples em casos de matrizes diagonais). A desvantagem desse método é a estabilidade estar em função do tamanho do incremento de tempo ${ }^{1}$, demandando passos pequenos e, portanto, muitas iterações.

Para fenômenos de impacto é desejado avaliar a resposta do sistema em curtos períodos de tempo, com pequenos intervalos de tempo. Dessa forma, a necessidade do pequeno incremento de tempo inviabilizaria a utilização do método implícito. Assim, a escolha do método explícito se torna inevitável. No método explícito, os valores de aceleração, ü, e velocidade, $\dot{u}$, são obtidos através do Método da Diferença Central (BATHE, 1996):

$$
\begin{aligned}
& \ddot{\mathbf{U}}=\frac{1}{\Delta t^{2}} \cdot\left({ }^{t-\Delta t} \mathbf{U}-2 \cdot{ }^{t} \mathbf{U}+{ }^{t+\Delta t} \mathbf{U}\right) e \\
& \dot{\mathbf{U}}=\frac{1}{2 \Delta t} \cdot\left(-{ }^{t-\Delta t} \mathbf{U}-2 \cdot{ }^{t} \mathbf{U}+{ }^{t+\Delta t} \mathbf{U}\right) .
\end{aligned}
$$

Considerando a Eq. 6.2a, escrita com a notação da matriz em função do instante $t$, $\mathbf{M}^{t} \ddot{\mathbf{U}}+\mathbf{C}^{t} \dot{\mathbf{U}}+\mathbf{K}^{t} \mathbf{U}={ }^{t} \mathbf{R}$, substituindo os termos das Eqs. 6.3a e 6.3b, obtém-se:

$$
\left(\frac{1}{\Delta t^{2}} \mathbf{M}+\frac{1}{2 \Delta t \mathbf{C}}\right)^{t+\Delta t} \mathbf{U}={ }^{t} \mathbf{R}-\left(\mathbf{K}-\frac{2}{\Delta t^{2} \mathbf{N}}\right)^{t} \mathbf{U}-\left(\frac{1}{\Delta t^{2}} \mathbf{M}-\frac{1}{2 \Delta t} \mathbf{C}\right)^{t-\Delta t} \mathbf{U}
$$

desprezando os termos relacionados ao amortecimento:

\footnotetext{
${ }^{1}$ Comumente conhecido em inglês como timestep, ou passo de tempo.
} 


$$
\left(\frac{1}{\Delta t^{2}} \mathbf{M}\right)^{t+\Delta t} \mathbf{U}={ }^{t} \mathbf{R}-\left(\mathbf{K}-\frac{2}{\Delta t^{2} \mathbf{N}}\right)^{t} \mathbf{U}-\left(\frac{1}{\Delta t^{2}} \mathbf{M}\right)^{t-\Delta t} \mathbf{U}
$$

Conforme comentado, a estabilidade da Eq. 6.5 - segundo o critério de Courant depende da escolha de um incremento de tempo inferior ao incremento de tempo crítico (WRIGGERS, 2008; ZIENKIEWICZ et al., 2005),

$$
\Delta t_{\text {crtico }} \approx \frac{h}{C_{L}}
$$

onde $h$ é a dimensão característica do menor elemento da malha e $C_{L}$ é a velocidade de propagação de uma onde de compressão. $C_{L}=\sqrt{E(1-\nu) /[\rho(1+\nu)(1-2 \nu)]}$ para um material isotrópico, para um módulo de elasticidade $E$. Assim, para diminuir os problemas de convergência, o valor do incremento de tempo a ser utilizado é uma redução do valor crítico, Eq. 6.6:

$$
\Delta t_{\text {crtico }} \leq \mu \frac{h}{C_{L}}, \quad \text { com } \quad \mu=[0.2 ; 0.9[
$$

sendo $\mu$, fator de redução do incremento de tempo crítico ${ }^{2}$. A escolha de $\mu$ depende de fatores como modelo de material e a própria modelagem em si.

\subsection{Escolha do Elemento}

A criação de um modelo em elementos finitos de um estrutura depende das dimensões, geometria, tipo de análise, material, carregamento etc. Parte dessa modelagem inclui a simplificação da geometria, escolha de material e tipo de elemento: treliças, vigas, plano, casca, sólido.

A geometria deste estudo possui algumas características relevantes:

I. Constituídas por chapas finas;

II. Flambagem;

III. Possível autocontato;

IV. Grandes deformações;

V. Possível falha. 


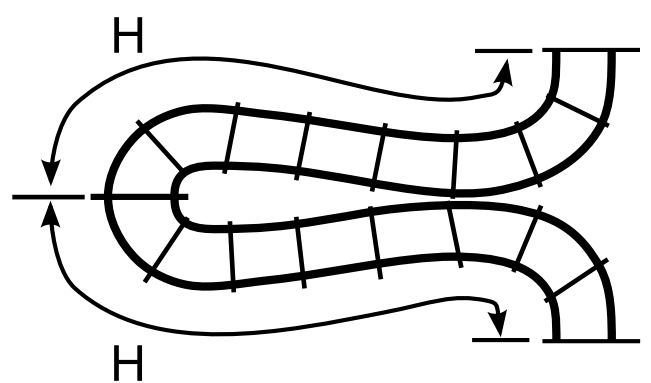

Figura 6.1 Número de elementos de dobra por flambagem. Adaptado de Paik et al. (2003).

Sobre $I$ - Chapas finas. Em geral, utiliza-se elementos de casca para a representação de estruturas construídas a partir de chapas finas. Através desses elementos se consegue menor custo computacional e simplificação na construção da malha (no presente caso). Contudo, tal representação é válida se a composição de esforços atuantes se aproximar do caso plano de tensão, i.e., tensão perpendicular ao plano desprezível. Em modelos de elementos finitos sólidos que representam estruturas sob flexão é recomendado o uso de, ao menos, quatro elementos ao longo da espessura; aumentando consideravelmente o número de elementos e reduzindo muito o tamanho mínimo desses elementos e, por conseguinte, o valor do incremento de tempo, conforme Eq. 6.7. Supostamente, para uma chapa de $3 \mathrm{~mm}$, o elemento deve ter, no mínimo, 0,75 mm ao longo da espessura.

Sobre II - Flambagem. Paik e Thayamballi (2003) ressalta que, para representar o comportamento da estrutura sob flambagem, são necessários ao menos oito elementos ao longo do comprimento de meia dobra, Fig. 6.1. Para a estrutura de estudo $(b=444 \mathrm{~mm}$ e $t=3 \mathrm{~mm})$.

$$
\begin{gathered}
H=1,103(b t)^{0,5}=40,3 \mathrm{~mm} \\
\therefore L_{\text {elemento }} \leq 5,03 \mathrm{~mm},
\end{gathered}
$$

resultando em uma relação entre espessura e tamanho do elemento é igual a 1,666. Caso haja necessidade de refinar a malha, o tamanho do elemento será ainda menor, provavelmente violando os preceitos de malha de casca ${ }^{3}$.

Sobre III - Autocontato. Em casos de dobras sucessivas é esperado que a estrutura, ao flambar, encoste em si mesma, transferindo esforços e resistindo de forma consistente.

\footnotetext{
${ }^{2}$ Referenciado no programa comercial de elementos finitos LS-Dyna como TSSFAC, Time Step Scaling Factor.

${ }^{3}$ Autores recomendam que a razão do tamanho de elemento e a espessura esteja entre $2 \sim 10$. Sendo pouco recomendável utilizar valores próximos ou menores que 1.
} 
Nessas situações, as malhas de casca possuem desvantagem por exigir do algoritmo a projeção ${ }^{4}$ da superfície superior e inferior, gerando instabilidades. Além disso, a compressão da chapa não proporcionará deformações ao longo da espessura, visto que a formulação - de casca - não contempla tais efeitos (estado plano de tensão). Em oposição a todas essas desvantagens, o elemento sólido é capaz de capturar todos esses efeitos.

Sobre $I V$ - Grandes Deformações. Algumas simulações foram realizadas de forma a verificar a correlação entre as curvas de força e deslocamento do ensaio de tração obtidas experimentalmente, com aquelas obtidas via elementos finitos com entrada $\sigma$ vs. $\varepsilon$. Nessas análises foi observado que elementos sólidos de integração seletiva não eram estáveis para grandes deformações, gerando erros e interrupção da solução. A recomendação nesses casos de grandes deformações é utilizar elementos de integração reduzida (LSTC, 2006, 2007), atentar à "irregularidade" dos elementos durante a simulação e refinar a malha (BATHE, 1996; ZIENKIEWICZ et al., 2005).

Sobre $V$ - Falha de Material. Em geral, modelos numéricos simples representativos de falhas de material carecem de malhas bastante refinadas de forma a capturar o efeito local, sem que subestimem os valores reais (ALSOS; AMDAHL; HOPPERSTAD, 2009). Assim, é desejado que, ao utilizar este critério de falha simplificada, o refino de malha seja suficiente para representar os ensaios.

Tabela 6.1 Comparativo entre elementos de casca e sólido para quesitos de interesse

\begin{tabular}{lcc}
\hline & Casca & Sólido \\
\hline Modela Chapas Finas & $\checkmark$ & $\checkmark^{1}$ \\
Flambagem & $\approx$ & $\checkmark$ \\
Autocontato & $\approx^{2}$ & $\checkmark$ \\
Grandes Deformações & $\checkmark^{3}$ & $\checkmark^{3}$ \\
Custo Computacional & $\checkmark^{3}$ & $\approx^{4}$ \\
Refino de Malha & $\approx^{5}$ & $\checkmark$ \\
Variação da Espessura & $\checkmark^{6}$ & $\checkmark$ \\
\hline
\end{tabular}

\footnotetext{
${ }^{1}$ Ao menos 4 elementos ao longo da espessura.

2 Instabilidade.

${ }^{3}$ Recomendado utilizar integração reduzida.

4 Bastante superior.

${ }^{5}$ Limitado pelo tamanho mínimo (relação com a espessura).

${ }^{6}$ Se escolhida formulação que considera cisalhamento. O resultado é estocado em variáveis do programa.
}

Os itens supracitados geraram a Tabela 6.1. Ambos os tipo de elementos são capazes

\footnotetext{
${ }^{4}$ Visto que somente a linha "neutra" é modelada.
} 
de modelar o fenômeno em questão. Entretanto, apesar do custo computacional maior, o elemento sólido permite maior flexibilidade de sua aplicação. Assim, sendo a estabilidade da solução e a capacidade de refino as principais preocupações, o painel duplo será modelado com elementos sólidos de 8 nós de tensão constante, com um ponto de integração ${ }^{5}$. Para contornar o problema de modos espúrios, foi escolhido o Controle de Hourglass de Rigidez $^{6}$ — ao invés do viscoso, devido às baixas velocidades envolvidas.

\subsection{Modelo Numérico}

Os modelos virtuais (em CAD), Fig. 6.2a das peças constituintes do painel duplo foram importados e malhados no programa de pré-processamento Altair Hypermesh 10.0. Todas as chapas foram modeladas com 4 elementos sólidos ao longo da espessura, como pode ser observado na Fig. 6.2b.

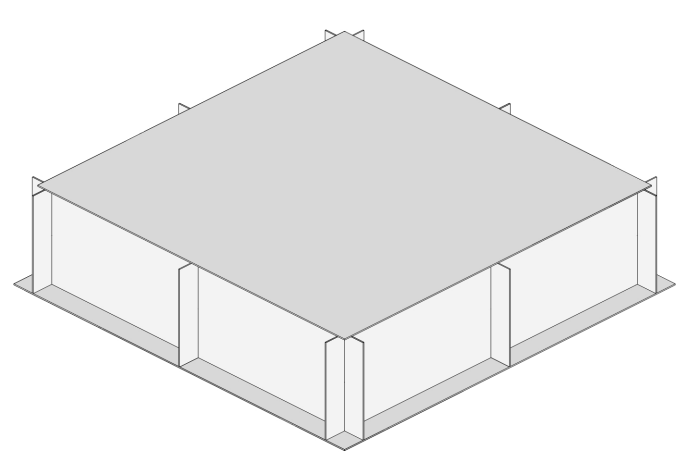

(a) Vista isométrica da geometria do painel duplo.

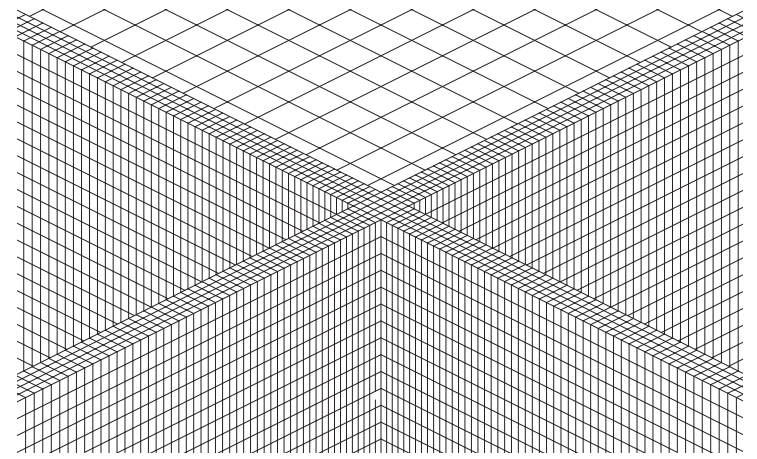

(b) Vista isométrica da junção cruciforme. Quatro elementos ao longo da espessura para todas as chapas.

Figura 6.2 Modelo numérico do painel duplo. Chapas foram modeladas com 4 elementos ao longo da espessura.

O tamanho do elemento variou no plano da chapa conforme a deformação esperada. Ao centro, na área cruciforme - esquerda da Fig. 6.3a - foi respeitada que a solicitação comprimiria os elementos na direção da altura do painel, enquanto que esticaria estas chapas lateralmente. Dessa forma, a largura e altura do elemento foram definidas tal que compensasse esse efeito de deformação, como pode ser observado na Fig. 6.3. Os elementos criados possuem o mínimo de 0,75 x 0,61 x 1,5 mm de espessura, largura e altura. Tal refino não se mostrava necessário para o restante da cruciforme, sendo razoável efetuar uma transição, Fig. 6.3b, para aumentar o tamanho dos elementos e diminuir o custo computacional. Transições de tamanhos de elemento também foram feitas nas chapas

\footnotetext{
${ }^{5} \mathrm{ELFORM}=1$, escolha padrão do programa LS-Dyna. É um elemento de baixo custo computacional que possui como contrapartida modos de vibrar espúrios, exigindo controle de rigidez ou "Controle de Hourglass" (LSTC, 2006, 2007).

${ }^{6} \mathrm{IHQ}=4$. Forma de rigidez de Flanagan-Belytschko (LSTC, 2006).
} 
superior e inferior, porém indiretamente, Fig. 6.4.

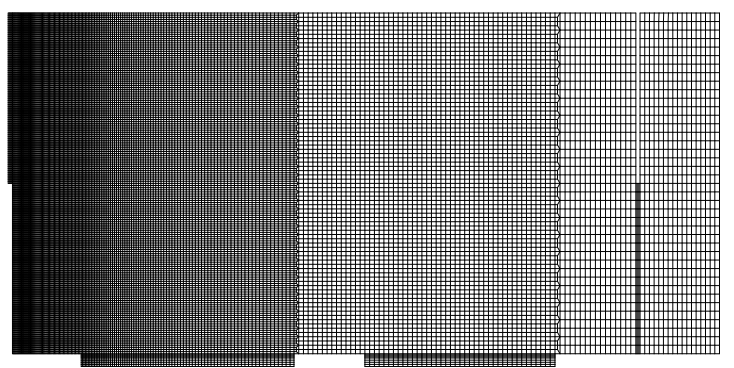

(a) Vista frontal, metade da chapa central.

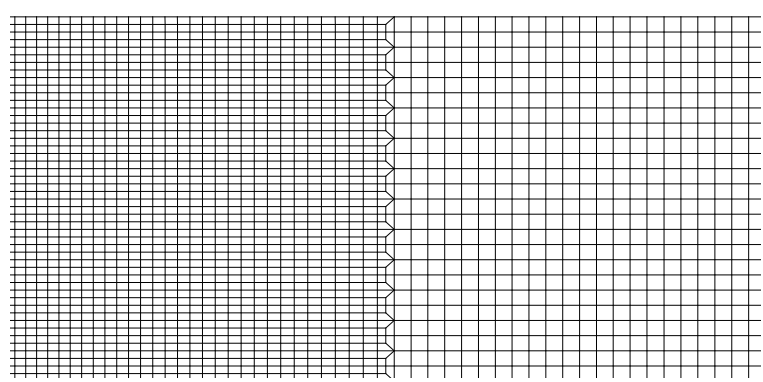

(b) Detalhe transição de elementos, reduzindo o número pela metade.

Figura 6.3 Transição do tamanho do elemento das chapas cruciformes.

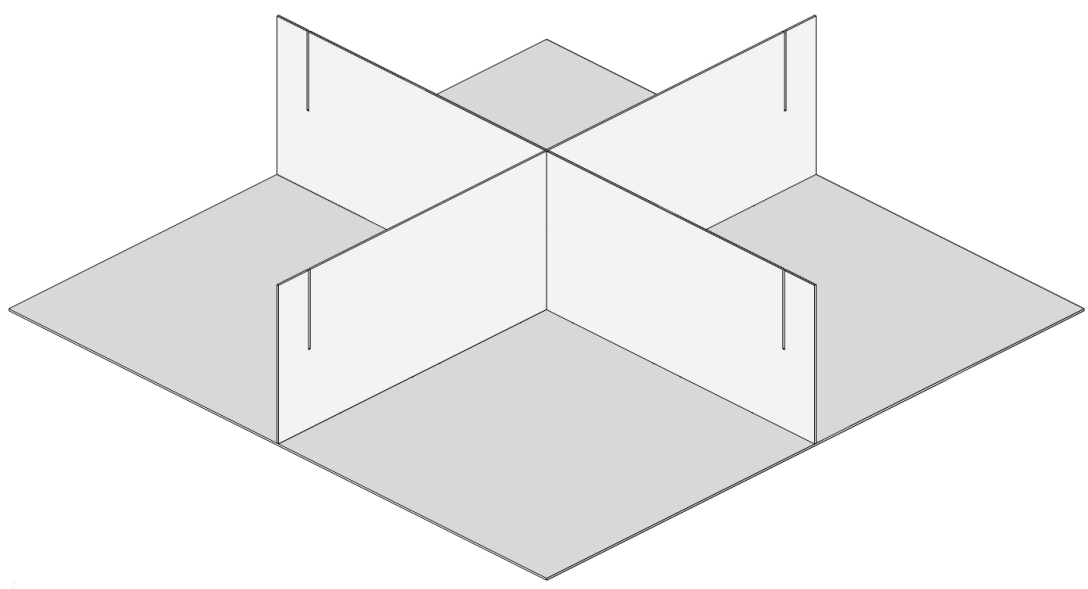

(a) Interseção Cruciforme em CAD.

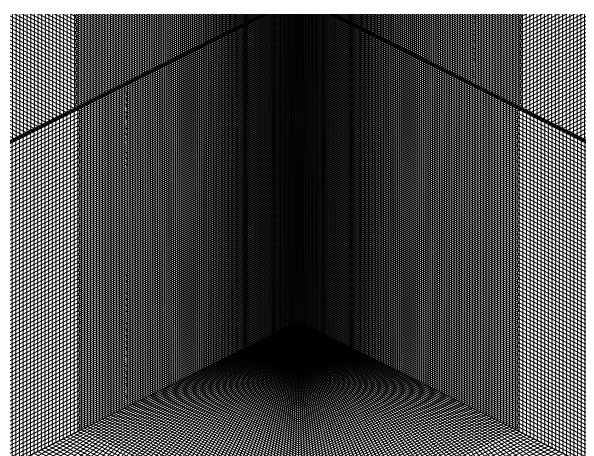

(b) Detalhe da malha da Interseção cruciforme e início da transição das chapas centrais.

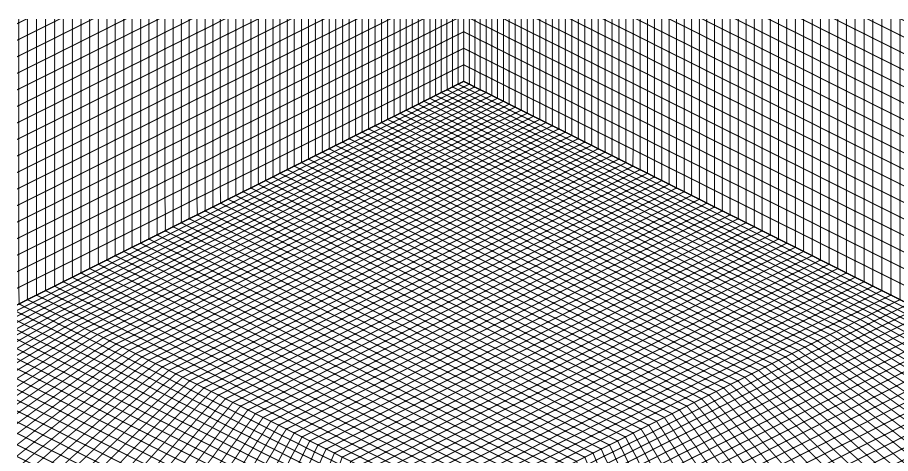

(c) Close-up da malha no centro da chapa. Elementos alongadas verticalmente e razão de aspecto quadrado nas chapas inferior e superior.

Figura 6.4 Detalhes da malha em elementos finitos para interseção em cruz.

As junções entre as chapas mantiveram a coincidência entre os nós, de modo a simplificar o acoplamento através do programa de elementos finitos. As laterais da chapa mantiveram elementos de tamanho igual àqueles das extremidades das cruciformes, sempre mantendo razão de aspecto menor que 10.

O suporte, Fig. 6.5a, foi simplificado e modelado com elementos sólidos, mantendo 
um pequeno raio interno para evitar problemas do algoritmo de contato; como pode ser observado no detalhe da Fig. 6.5b. A bigorna também foi modelada de forma simplificada, tendo somente a altura de $0,5 \mathrm{~m}$, Fig 6.6. Quanto ao indentador, foi modelado também com elementos sólidos, com refino acentuado para representar melhor a superfície complexa e, portanto, o contato com os elementos do painel duplo, Fig. 6.7.

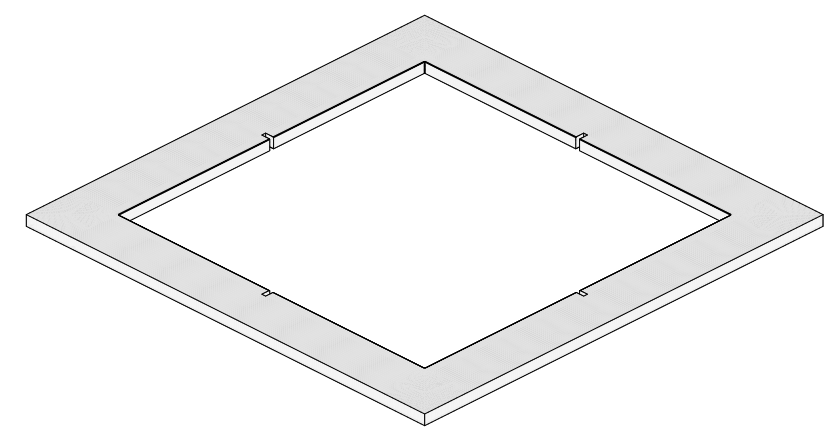

(a) Suporte representado numericamente.

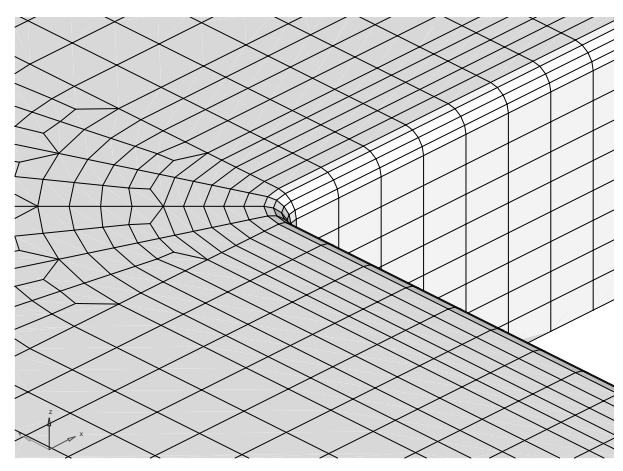

(b) Detalhe do raio interno do suporte e transição do refino de malha do canto esquerdo.

Figura 6.5 Geometria e malha em elementos finitos do suporte.

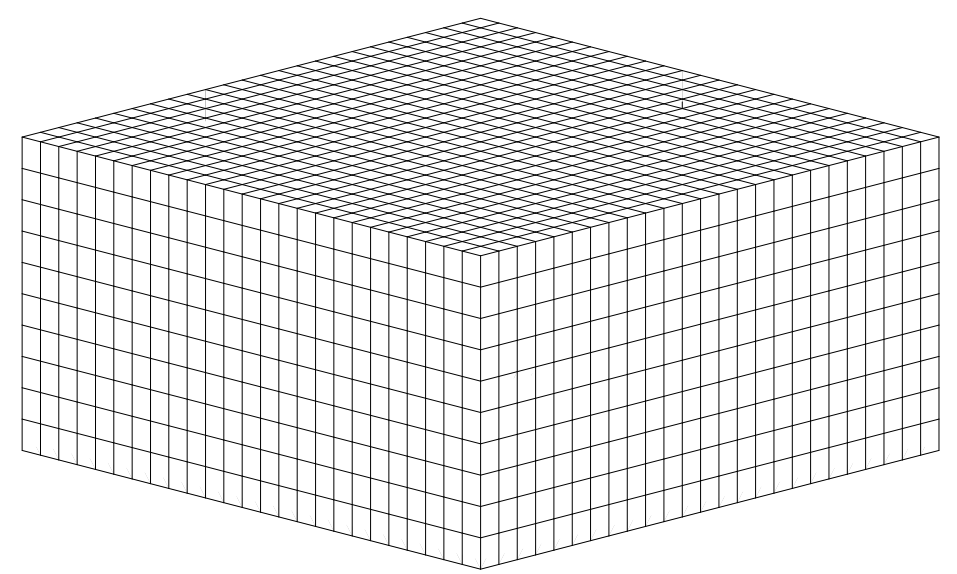

Figura 6.6 Malha em elementos finitos da bigorna, representada com 0,5m de altura e 1,0x1,0m. Tamanho médio do elemento: $40 \mathrm{~mm}$.

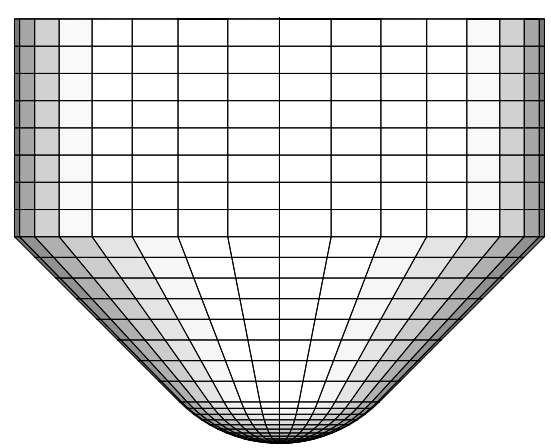

(a) Vista lateral.

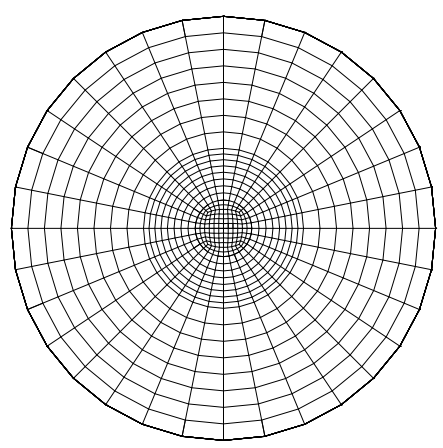

(b) Vista inferior.

Figura 6.7 Malha em elementos finitos do Indentador. 
Conforme citado anteriormente, existe a possibilidade de autocontato das chapas do painel duplo. O programa LS-Dyna possui a opção (cartão ou keyword) de contato de uma única superfície ${ }^{7}$, a qual define o contato entre elementos, mesmo que pertençam a um mesmo componente. Assim sendo, foi atribuída essa propriedade ao grupo formado pelos elementos das chapas do painel e dos elementos do suporte. A interface suportebigorna foi modelada a partir da superfície superior da bigorna ${ }^{8}$ e de todos os elementos sólidos do suporte ${ }^{9}$, com o contato entre superfícies ${ }^{10}$. Este cartão também foi atribuído à interface indentador-painel, na qual os elementos do indentador comandam o contato e os elementos da chapas superior e pertencentes à cruciforme servem como comandadas.

Tabela 6.2 Cartões de modelos de materiais utilizados na análise resolvida pelo programa LS-Dyna.

\begin{tabular}{|c|c|c|c|c|c|c|}
\hline & $\begin{array}{c}\rho \\
{\left[10^{-9} \text { ton } / \mathrm{mm}^{3}\right]}\end{array}$ & $\begin{array}{c}E \\
{\left[10^{3} \mathrm{MPa}\right]}\end{array}$ & $\begin{array}{c}\nu \\
{[-]}\end{array}$ & $\begin{array}{c}\sigma_{0} \\
{[\mathrm{MPa}]}\end{array}$ & $\begin{array}{c}p \\
{[-]}\end{array}$ & $\begin{array}{c}C \\
{[1 / \mathrm{s}]}\end{array}$ \\
\hline MAT_ELASTIC & 7,85 & 210 & 0,3 & - & - & - \\
\hline MAT_RIGID & -1 & 210 & 0,3 & - & - & - \\
\hline MAT_ $24^{2}(0,25 \mathrm{~mm})$ & 7,85 & 180 & 0,3 & 282.0 & 5,188 & 42,57 \\
\hline MAT_ $24^{2}(3,00 \mathrm{~mm})$ & 7,85 & 97.9 & 0,3 & 182.7 & 5,188 & 42,57 \\
\hline
\end{tabular}

${ }^{1}$ Ajustado para resultar na massa de impacto. $\rho=6.61830 \times 10^{-7}$ ton $/ \mathrm{mm}^{3}$, para $1000 \mathrm{~kg}$ no protótipo.

${ }^{2}$ Pode ser definido também como *MAT_PIECEWISE_LINEAR_PLASTICITY.

Para os componentes acessórios como bigorna e suporte, foram atribuídas propriedades elásticas do aço, usando o modelo de material linear elástico ${ }^{11}$; quanto ao indentador, foi utilizado modelo rígido ${ }^{12}$ — conforme Tabela 6.2. Já os elementos do painel, foram modelados com o comportamento elastoviscoplástico isotrópico, e cuja curva tensão vs. deformação plástica, Fig. 6.8, foi inserida no programa ${ }^{13}$ com as propriedades das Tabelas B.1 e B.2, do Apêndice B. Por fim, na Tabela 6.3 encontram-se as condições iniciais dos ensaios experimentais e as entradas do programas. Devido ao erros de arredondamento, a massa do indentador na simulação é próxima ao valor desejado, porém não idêntica. Na próxima seção os resultados da simulação são apresentados e discutidos.

\footnotetext{
${ }^{7 *}$ CONTACT_AUTOMATIC_SINGLE_SURFACE (LSTC, 2007).

${ }^{8}$ Elementos de casca, representativos da superfície superior. Atuando como Slave ou comandada.

${ }^{9}$ Atuam como Master. Comandando o contato por possuir malha mais refinada.

$10 *$ CONTACT_AUTOMATIC_SURFACE_TO_SURFACE (LSTC, 2006).

$11 *$ MAT ELA $\overline{S T I C}$ ou *MAT 03 .

$12 *$ MAT_RIGID ou *MAT_20. Apesar de ser rígido, o programa necessita de um valor do módulo de elasticidade para realizar alguns cálculos.

$13 *$ MAT_PIECEWISE_LINEAR_PLASTICITY ou *MAT_24.
} 


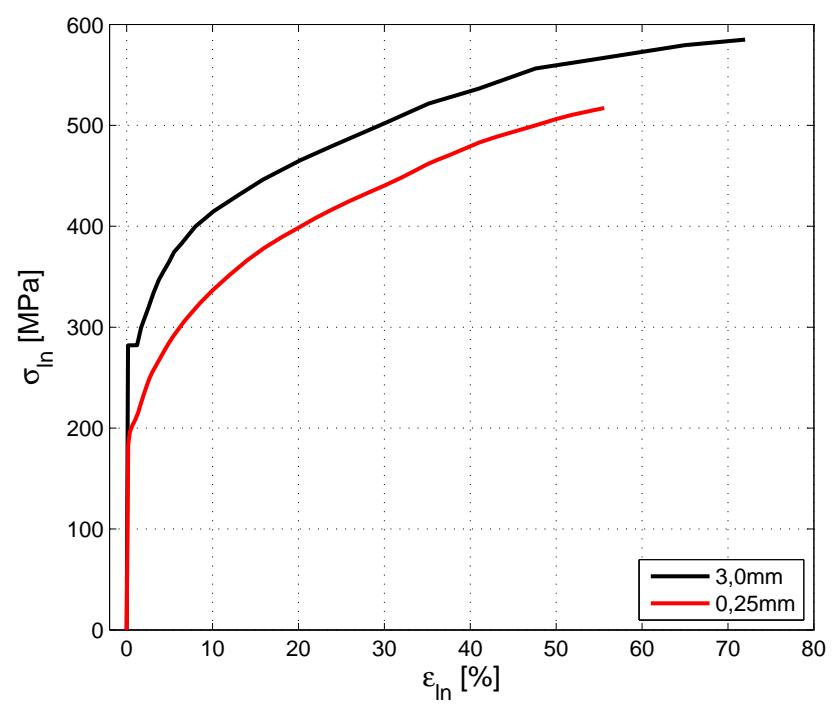

Figura 6.8 Curva tensão verdadeira vs. tensão verdadeira utilizadas no programa de elementos finitos.

Tabela 6.3 Comparativo entre condições do ensaio experimental e as entradas do programa em elementos finitos.

\begin{tabular}{lccccc}
\hline & & \multicolumn{2}{c}{$\mathrm{G}[\mathbf{K g}]$} & \multicolumn{2}{c}{$V_{0}[\mathbf{m} / \mathbf{s}]$} \\
& Escala & Num. & Exp. & Num. & Exp. \\
\hline Protótipo & $1: 1$ & 998,1 & 999,4 & - & - \\
MLT & $1: 12$ & 0,587 & 0,588 & 7,0 & 7,065 \\
VSG & $1: 12$ & 0,699 & 0,698 & 5,6 & 5,67 \\
\hline
\end{tabular}

\subsection{Resultados}

Nos dois capítulos anteriores foram mostrados resultados dos ensaios experimentais e aqueles obtidos através de equacionamento analítico. Nesta seção, esses resultados serão comparados aos obtidos através de simulação para cada painel, além de serem correlacionados entre si com o uso das leis de similaridade.

A solução dos modelos criados em elementos finitos, com o total de 2.078 .861 nós e 1.732.678 elementos, levou cerca de sete dias, para cada modelo virtual. Como resultado o programa fornece imagens de estado periódicas, além das variáveis de estado do indentador como tempo, posição, velocidade e aceleração. Este último resultado, multiplicado pela massa definida a priori, fornece os resultados de força. Estes, integrados ao longo do deslocamento — saída do programa - resulta na energia dissipada do indentador.

Os resultados numéricos para o protótipo mostram que o formato da curva $F$ vs. $\delta$, Fig. 6.9a, do início até $\delta=0,03 \mathrm{~m}$, correlaciona bem tanto com o analítico como com o 
experimental. A partir desse ponto, os valores da curva numérica, em vermelho, ficam entre $20 \%$ e $30 \%$ abaixo da curva analítica (verde), e acima do que pode ser considerado uma porção falha do ensaio experimental. Da mesma forma, os resultados de Energia, Fig. 6.9b, seguem a mesma linha, ficando a curva numérica entre a analítica - mais rígida — e a experimental. A variação é de, no máximo, $30 \%$ entre a numérica e analítica e $20 \%$ entre numérica e experimental, para o mesmo deslocamento.

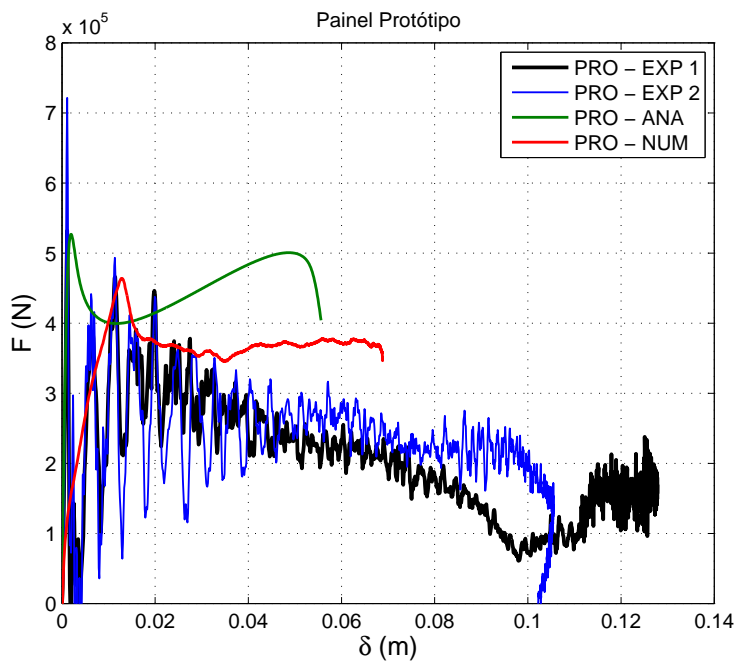

(a) Força vs. Deslocamento.

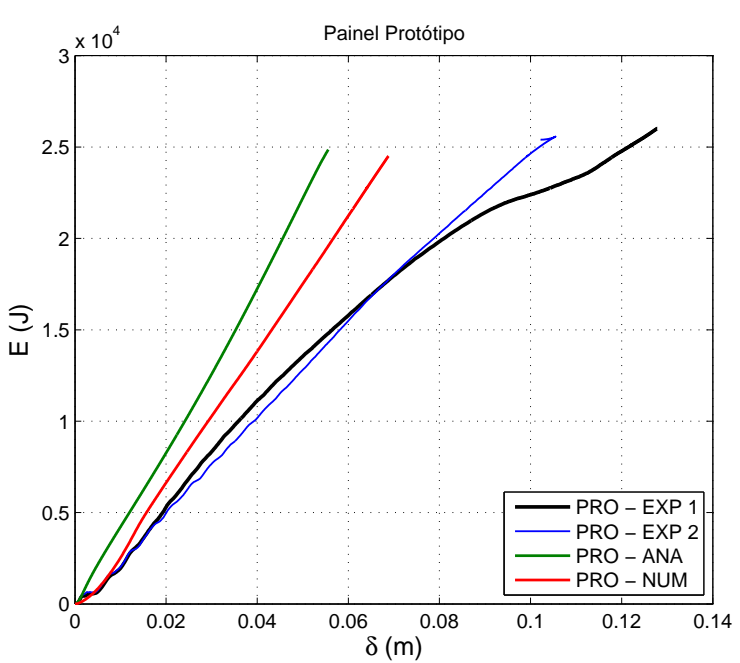

(b) Energia vs. Deslocamento.

Figura 6.9 Correlação entre resultados experimentais, analíticos e numéricos para o painel protótipo.

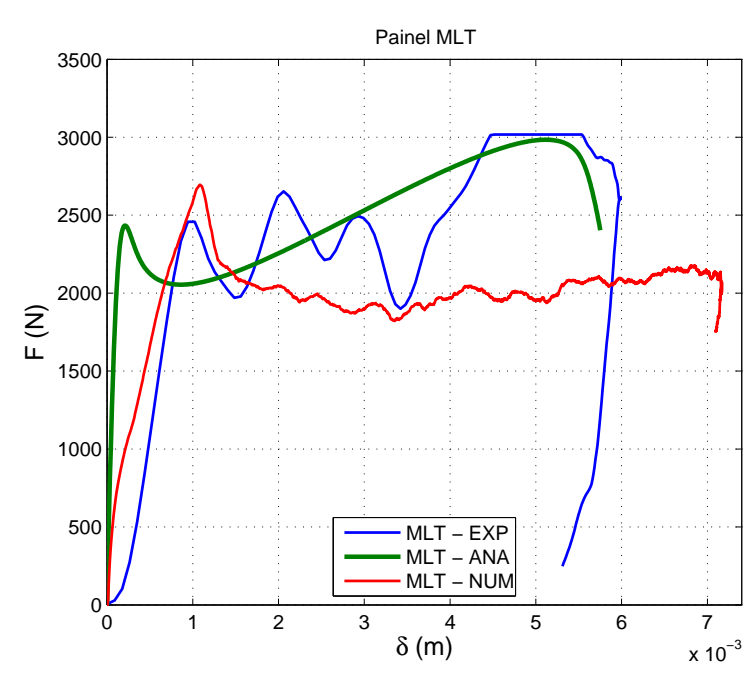

(a) Força vs. Deslocamento.

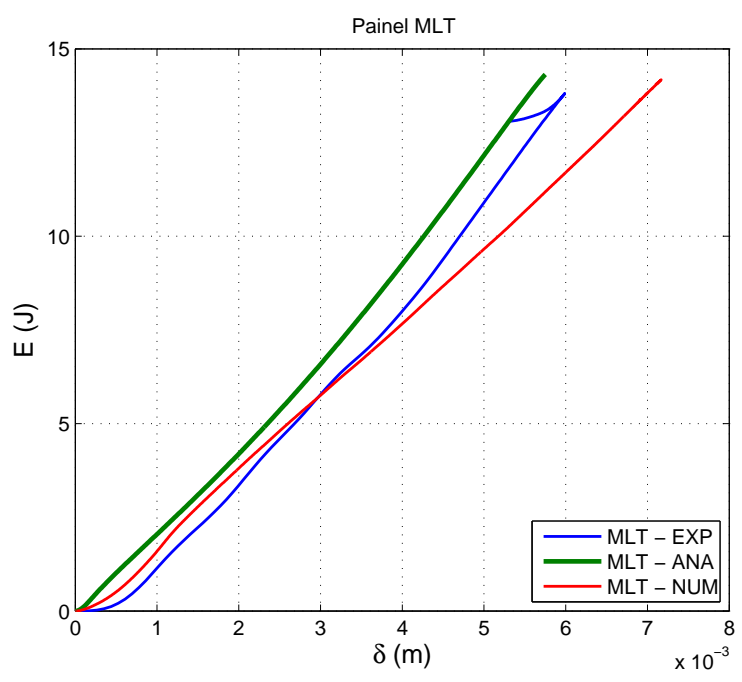

(b) Energia vs. Deslocamento.

Figura 6.10 Comparativo entre resultados experimentais, analíticos e numéricos para o ensaio do modelo MLT.

Com relação aos deslocamentos máximos, o analítico se mostrou mais rígido que o numérico, $\operatorname{com} \delta$ cerca de $0,02 \mathrm{~m}$ menor. Entretanto, se deslocada a curva analítica de modo a compensar a subida elástica, os deslocamentos máximos se aproximam muito. 


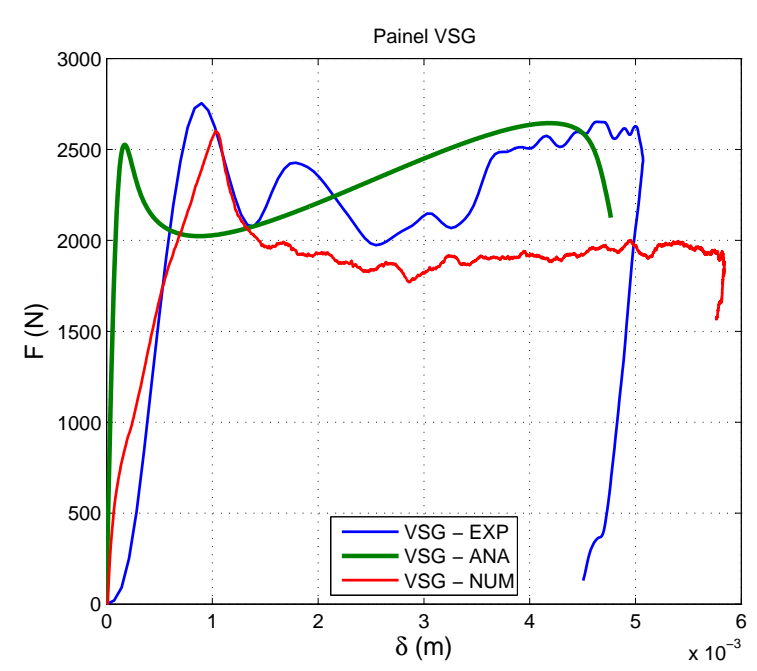

(a) Força vs. Deslocamento.

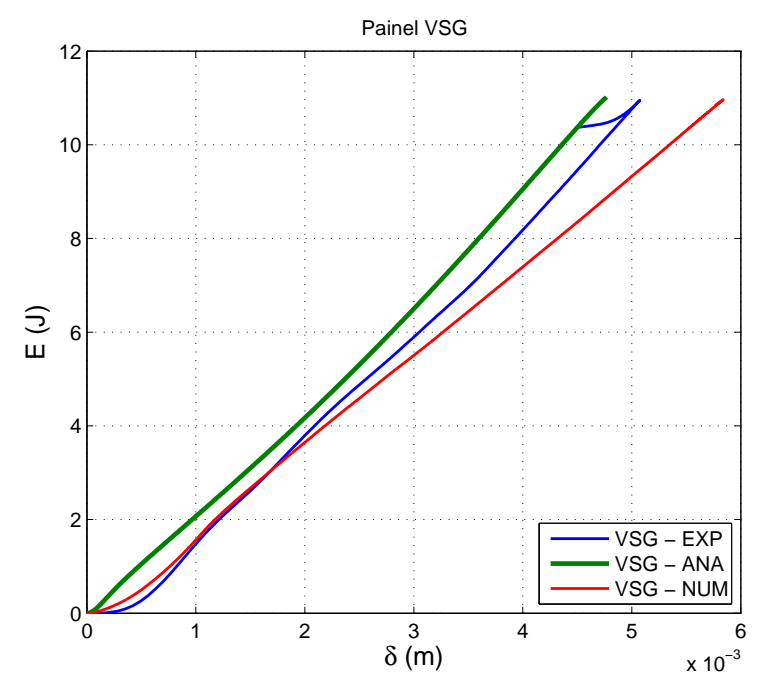

(b) Força vs. Deslocamento.

Figura 6.11 Comparativo entre resultados experimentais, analíticos e numéricos para o ensaio do modelo VSG.

O mesmo ocorre para a curva de energia analítica, a qual é paralela à numérica apenas deslocada do início.

Os resultados numéricos obtidos para o painel MLT, Fig. 6.10a, possuem forma similar ao do protótipo, Fig. 6.9a, sendo igualmente menos rígidos que os analíticos e experimentais. Importante notar que o resultado numérico capta somente parte da subida que ocorre a partir de $\delta=3,5 \mathrm{~mm}$ tanto para o analítico como para o experimental. Ainda, por ser menos rígido, atinge deslocamento maior que as outras curvas. Tal afirmação é observável na Fig. 6.10 b, curvas de $E$ vs. $\delta$ na qual a inclinação da curva obtida por elementos finitos é menor.

Também, a curva numérica (em vermelho), segue a experimental até $\delta=2 \mathrm{~mm}$, onde a partir desse valor decai de inclinação se comparada às demais. Isto apenas reflete a tendência observada na curva de $F$ vs. $\delta$, Fig. 6.10a, quando se cruzam. Por fim, os níveis de energia são adequados, como se pode esperar. De um aspecto geral, a simulação capta boa parte do comportamento observado no experimento.

A Fig. 6.11a mostra as curvas dos resultados obtidos para o painel VSG, bastante similares àquelas do MLT; com deslocamento máximo numérico cerca de $20 \%$ maior que o experimental (azul) e o analítico (verde). Também não se observa a tendência de subida ocorrida no experimento e o nível de força é inferior ao experimental a partir de $\delta=1,5 \mathrm{~mm}$. Isto reflete na curva de $E$ vs. $\delta$, Fig. $6.11 \mathrm{~b}$, na qual a numérica se distancia da experimental.

A comparação entre os resultados numéricos é realizada de forma similar ao feito para 
as curvas analíticas. Os resultados de força são corrigidos pelo fator $\beta_{F}$, a energia por $\beta_{E}$ e o deslocamento por $\beta$; gerando os gráficos $F$ vs. $\delta$, Fig. 6.12a e $E$ vs. $\delta$, Fig. 6.12b.

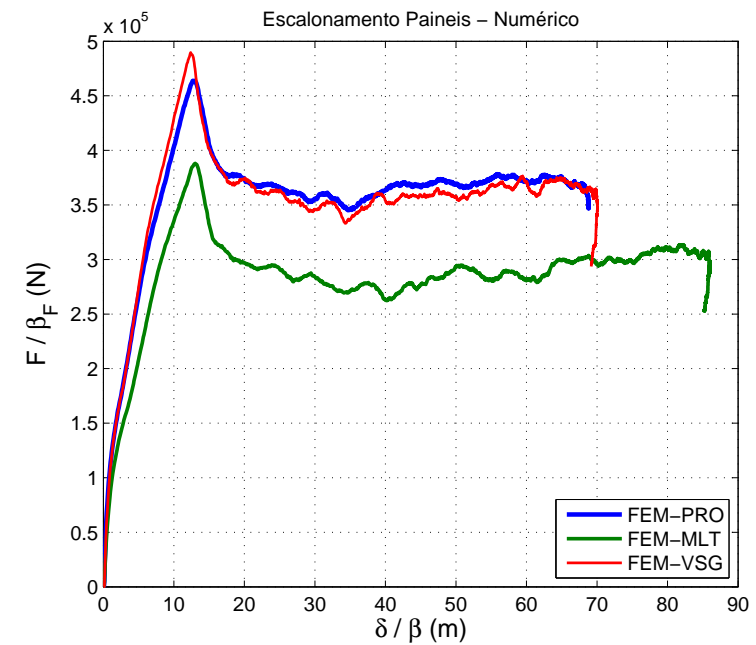

(a)

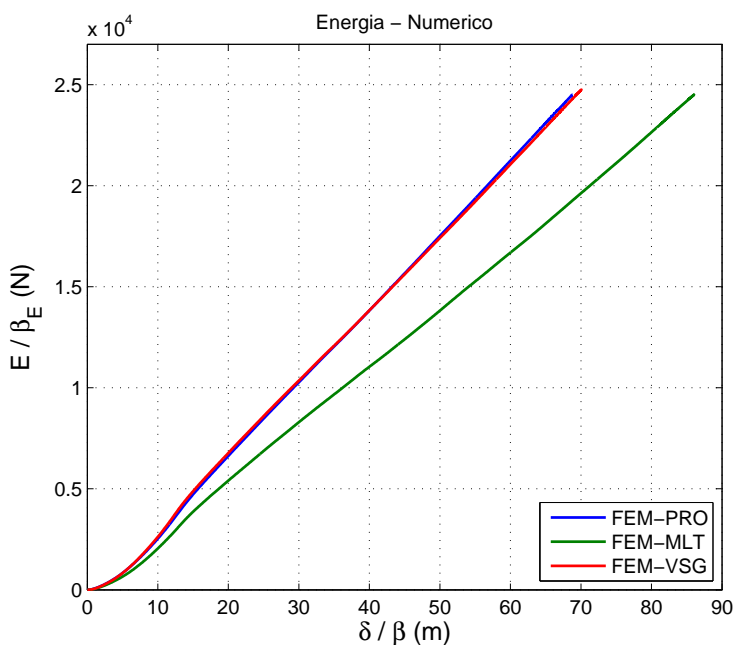

(b)

Figura 6.12 Correlação de resultados numéricos para os paineis Protótipo, MLT e VSG.

No primeiro deles é possível observar que o modelo VSG correlaciona bem com o protótipo, tanto em deslocamento máximo como para o nível de força. Contudo, é possível notar que o pico da curva VSG, além de ser 5\% maior, ocorre para um deslocamento levemente menor, o qual não ocorre para o MLT. Sobre este, não só o deslocamento máximo é cerca de $20 \%$ maior, como o nível de força é $20 \%$ menor que as duas outras curvas. Ao observar as Figs. 6.10a e 6.11a, nota-se que os deslocamentos do MLT são maiores que o do VSG, assim como os resultados de força. Destarte, as diferenças que surgem na Fig. 6.12a — do VSG ser maior que o MLT — advém dos fatores de escala de força $\beta_{F}$ que levam os resultados VSG mais próximos do Protótipo do que o MLT. Quanto aos deslocamentos, estão sujeitos ao mesmo fator $\beta$, então mantendo a proporção anteriormente vista.

As curvas de energia, Fig. 6.12b, refletem as curvas de $F$ vs. $\delta$; onde a curva de energia do VSG se superpõe à do protótipo. O mesmo não ocorre para o MLT, o qual possui valor máximo de energia próximo às demais, entretanto com deslocamento $20 \%$ maior.

Como comentado no início desta seção, os resultados numéricos não se limitam à resposta em termos de deslocamento, velocidade etc. do indentador, mas compreende, também, o estado de deformação, tensões etc. da estrutura calculado para intervalos de tempo definidos pelo usuário. Para os modelos serem mais facilmente comparáveis ao protótipo, além do tempo da simulação dos modelos, foi acrescida a equivalência em tempo do protótipo. Ou seja, a escala de tempo foi corrigida pelo fator $\beta_{t}$ correspondente 
tanto para o modelo VSG como para o MLT. À esquerda das Figs. 6.15 e 6.16 encontramse as imagens igualmente espaçadas em sequência da simulação do protótipo, e à direita, as do modelo MLT para a primeira figura e VSG, para a segunda. As imagens finais representam o ponto de inflexão, i.e., o momento em que a velocidade atinge zero. De modo a facilitar a visualização da interseção cruciforme, somente parte da opacidade da chapa superior foi mantida.

Nota-se na Fig. 6.15 que além de a escala de tempo ser diferente, $t=19,3$ ms para o protótipo contra $t / \beta_{t}=24,0 \mathrm{~ms}$ para o MLT, a aparência de deformação no MLT é maior que no protótipo. Fato este observável tanto na aparência da dobra como na sombra formada pela iluminação da chapa superior. Ainda sobre a dobra, a do MLT é simétrica para o sentido contrário ao ocorrido para o protótipo, o que acarreta apenas diferenças estéticas.

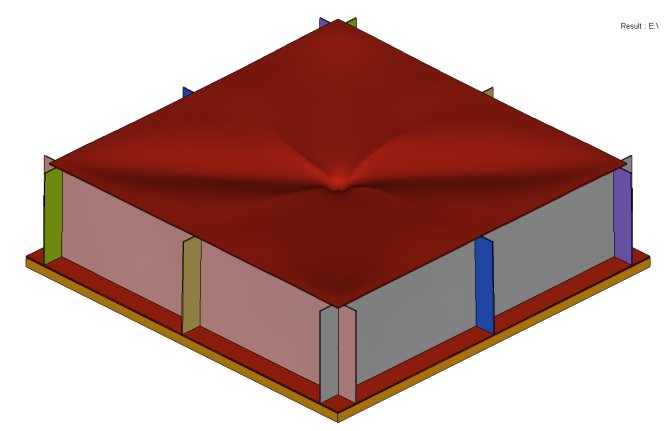

(a) Painel MLT ao fim da análise numérica por elementos finitos.

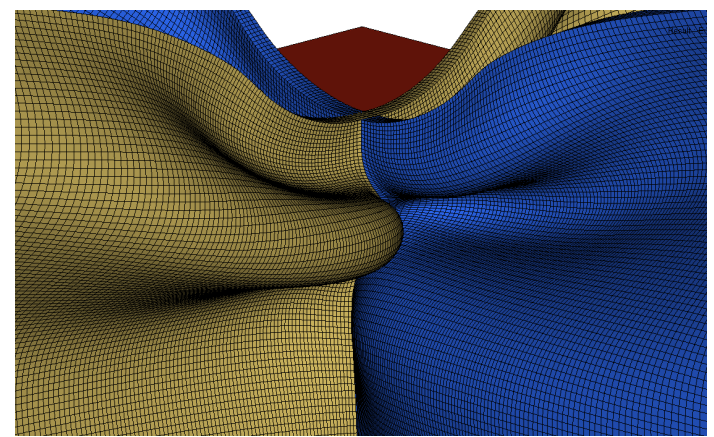

(b) Painel MLT sem a chapa superior. Autocontato entre os elementos da cruciforme.

Figura 6.13 Aspecto geral de deformação dos paineis simulados.

Para a Fig. 6.16, o tempo final da simulação do VSG é bem mais próximo do protótipo do que o ocorrido para o MLT, um indício de que o tempo corrigido representa bem o tempo do protótipo. Ademais, figura-a-figura é possível observar bastante similaridade no aspecto de deformação, sendo difícil enxergar pontos drasticamente diferentes, como se observa na Fig. 6.15. Isso caracteriza a qualidade de conseguir obter aspecto e deformações similares a um protótipo, com o uso de modelos. Ademais, como se pode notar nas Figs. $6.16 \mathrm{i}$ e $6.16 \mathrm{j}$, a dobra em forma de pétala, ou catavento, que ocorreu no mesmo sentido para o protótipo e VSG, algo sem maiores explicações ou comentários.

O aspecto geral da deformação foi similar nos três paineis virtuais simulados e de acordo com o mecanismo de deformação estipulado na premissa do Capítulo 5. É possível notar na Fig. 6.13b o autocontato que norteou a escolha de malha, bem como o estiramento dos elementos nessa região crítica, ambos de maneira similar aos paineis MLT e VSG, Fig. 4.17. Além disso, o desenho de deformação da chapa superior após o ensaio 
experimental, Figs. 4.17a e 4.17b é similar ao obtido em todas as simulações, Fig. 6.14. Tal comparação entre simulação e experimento não é possível para os paineis protótipos devido à falha ocorrida em ambos os ensaios. Apesar disso, a tendência de deformação da chapa superior no experimento dos protótipos e modelos, Fig. 4.6 e 4.13 guardam relação com a obtida numericamente, Fig. 6.13a.

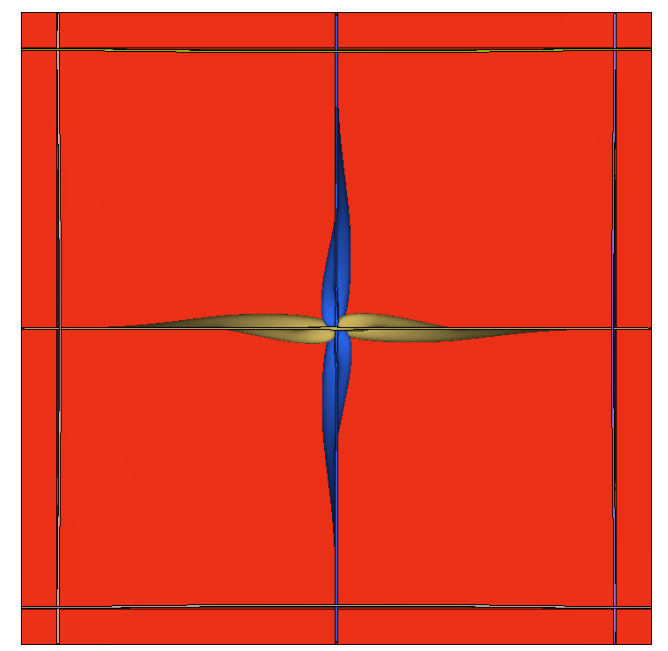

(a) Vista superior.

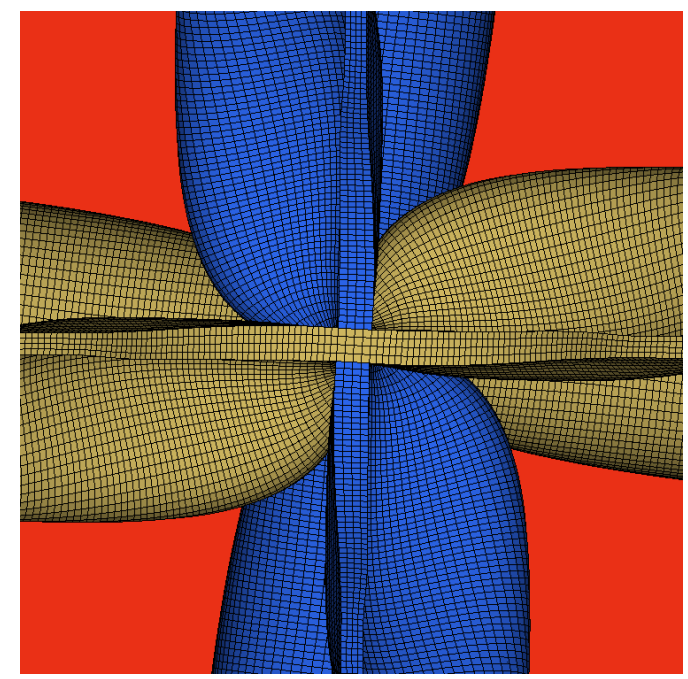

(b) Close no centro da cruciforme.

Figura 6.14 Vista superior do painel MLT simulado. Visualização sem a chapa superior. O mecanismo de flambagem da cruciforme ocorre em pétalas rotacionadas como um catavento ou moinho. 


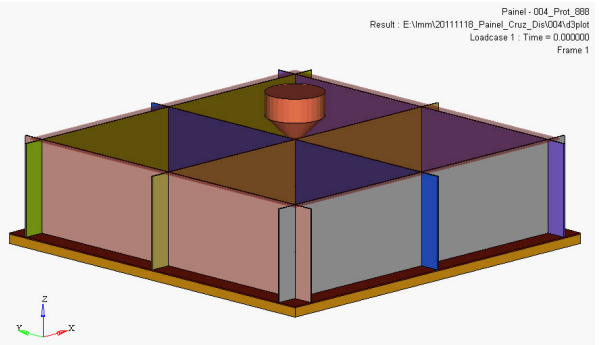

(a) $t=0$

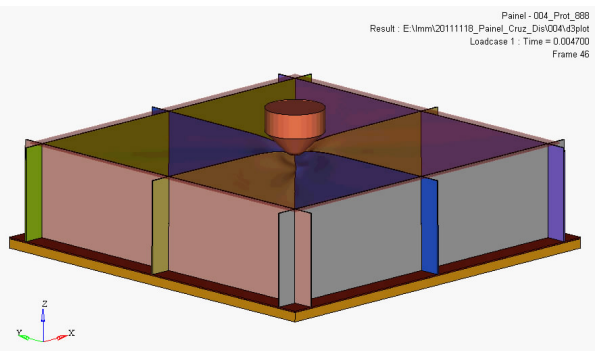

(c) $t=4,7$

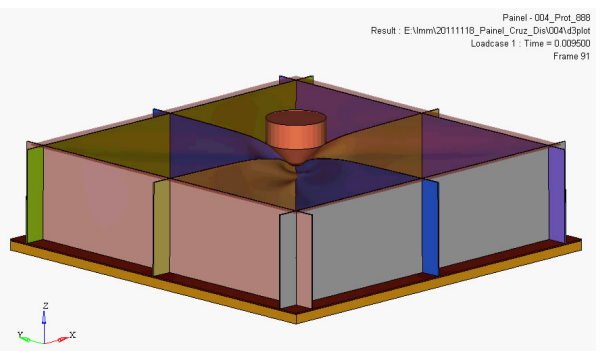

(e) $t=9,5$

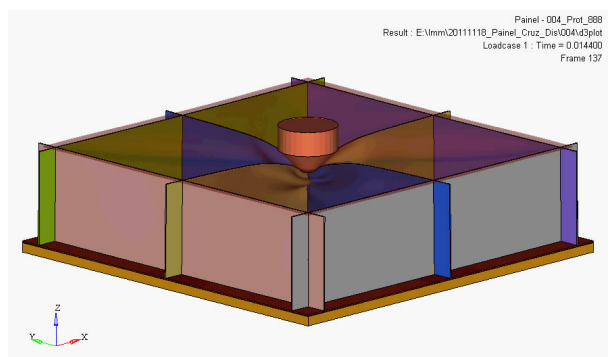

(g) $t=14,4$

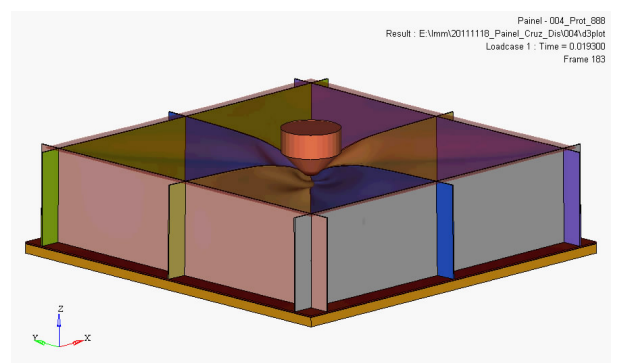

(i) $t=19,3$

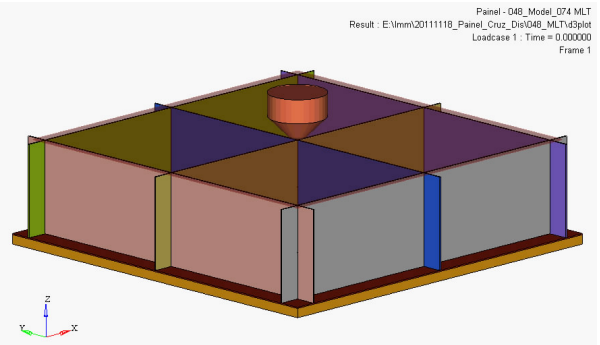

(b) $t=0$

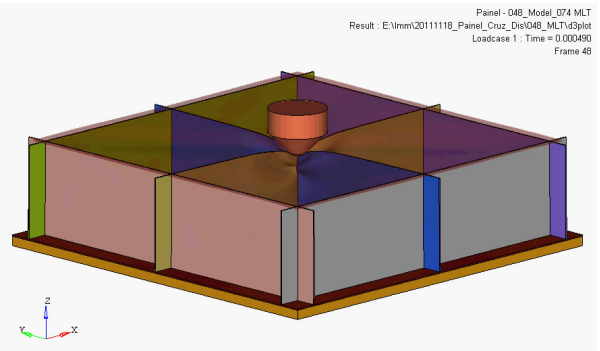

(d) $t=0,49$ ou $t / \beta_{t}=5,89$

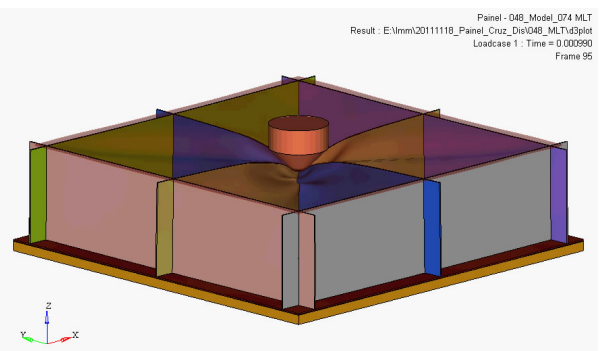

(f) $t=0,99$ ou $t / \beta_{t}=11,9$

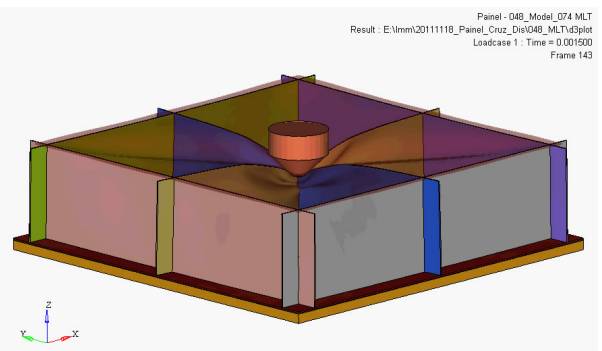

(h) $t=1,5$ ou $t / \beta_{t}=18,0$

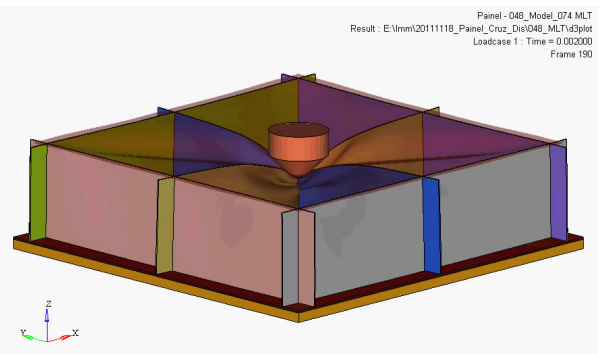

(j) $t=2,0$ ou $t / \beta_{t}=24,0$

Figura 6.15 Captura de quadros do vídeo da simulação numérica dos paineis Protótipo (à esquerda) e MLT (à direita). Instantes de tempo em milissegundo. Os tempos do modelo MLT foram corrigidos usando a fórmula $\beta_{t}=\beta$. 


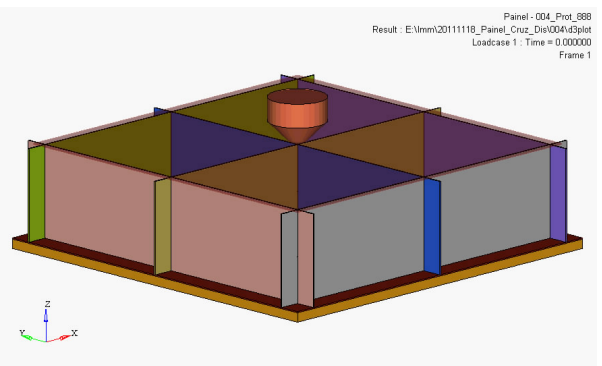

(a) $t=0$

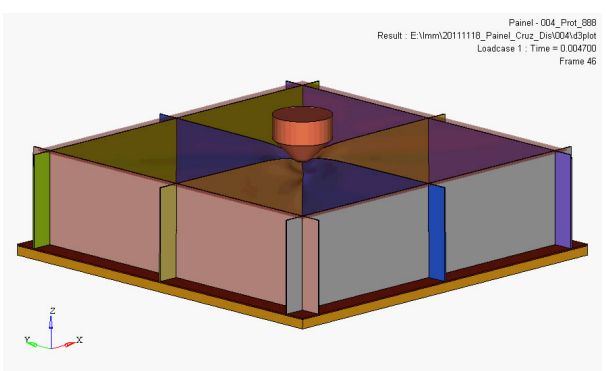

(c) $t=4,7$

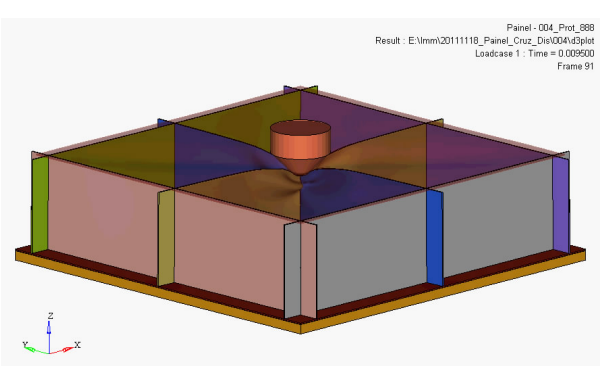

(e) $t=9,5$

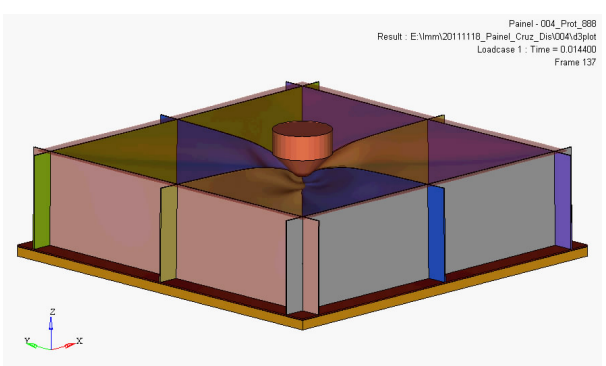

(g) $t=14,4$

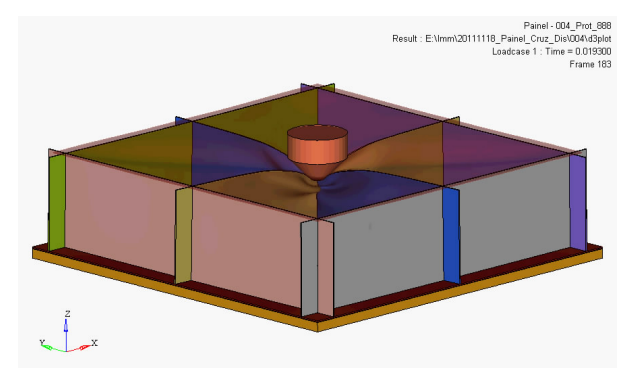

(i) $t=19,3$

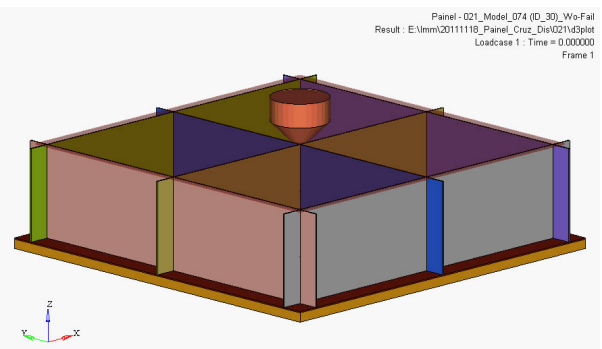

(b) $t=0$

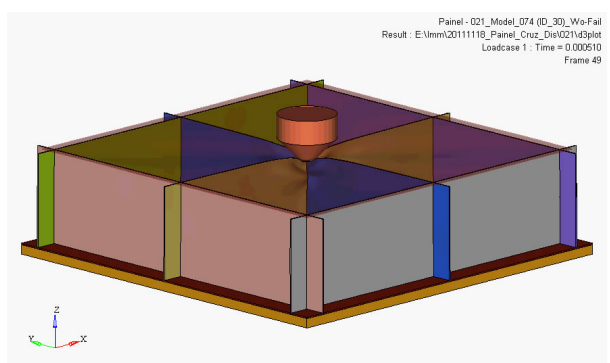

(d) $t=0,51$ ou $t / \beta_{t}=4,90$

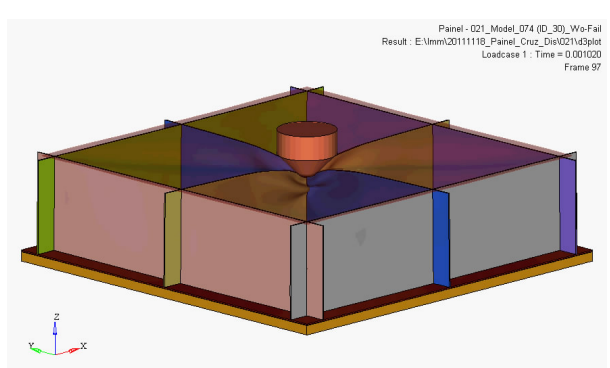

(f) $t=1,02$ ou $t / \beta_{t}=9,79$

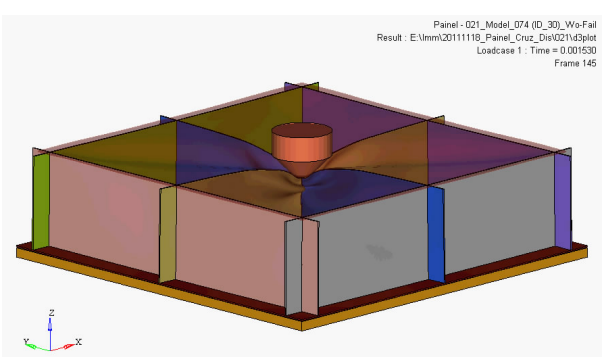

(h) $t=1,53$ ou $t / \beta_{t}=14,7$

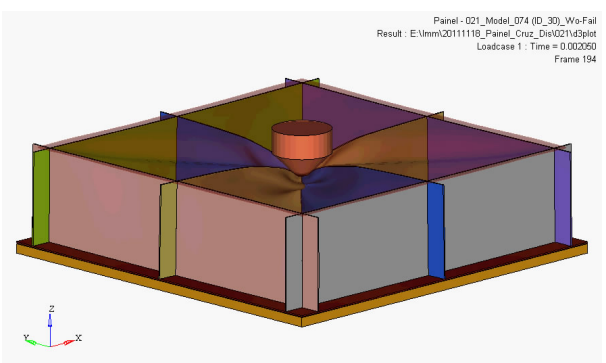

(j) $t=2,05$ ou $t / \beta_{t}=19,7$

Figura 6.16 Captura de quadros do vídeo da simulação numérica dos paineis Protótipo (à esquerda) e VSG (à direita). Instantes de tempo em milissegundo. Os tempos do modelo VSG foram corrigidos usando a fórmula $\beta_{t}=\beta / \beta_{V}$. 



\section{CAPÍtulo 7 \\ Comentários Finais}

Neste capítulo são comentadas de forma complementar as questões que emergiram durante o decorrer deste trabalho. Esses tópicos têm como base resultados mostrados nos capítulos anteriores, pontuando possíveis soluções para problemas enfrentados. Adicionalmente, são discutidas extensão e aprimoramento da técnica de escalonamento. Por fim, são plotadas as curvas força vs. deslocamento e energia vs. deslocamento dos paineis protótipo e modelos para as abordagens analítica, numérica e experimental.

\section{Força Média}

A comparação dos resultados analíticos e numéricos de força vs. deslocamento, deslocamento vs. tempo e Energia vs. deslocamento ou tempo, é realizada mais facilmente do que a dos resultados experimentais. Enquanto nas duas primeiras é possível estabelecer a quantidade de pontos a serem calculados e gravados, na última, isso é função da limitação do sistema de aquisição utilizado, em confronto com as características do ensaio como duração do evento, fundo de escala e a própria oscilação da resposta.

Uma forma de contornar a resposta oscilatória que dificulta a comparação é calcular a força média. Entretanto, o dado de força média do ensaio somente é significante se obtido a partir de um resultado experimental que proporcionou ao menos 2 dobras completas, já que varia bastante também no período de uma dobra. Isso posto, para a estrutura do presente trabalho, cálculos preliminares indicaram que solicitá-la a um deslocamento maior que uma dobra resultaria em falha, algo não contemplado pelas leis de similaridade aqui utilizadas. Além disso, dificultaria a preparação do ensaio, seja por um aumento de massa, seja pelo aumento da velocidade. 


\section{Experimento em Escala}

Uma das grandes preocupações em utilizar uma estrutura complexa é o modo de construila. Embora muitas fossem as ideias, não houve saída senão utilizar soldagem para construir os paineis. Na realidade, pode-se dizer que a influência da solda é importante já que altera a propriedade do material de forma diferente para o protótipo e modelo. Se houver método construtivo no qual não haja mudança nas características do material, este certamente poderá ser utilizado para correlacionar estruturas. Entretanto, o observado nos experimentos do Capítulo 4 é a influência da solda no painel protótipo, não ocorrendo o mesmo para o modelo utilizado para representá-lo. Sendo assim, se houver forma de causar o mesmo "dano" nas regiões próximas das soldas do painel protótipo, no modelo, certamente o ensaio seria mais esclarecedor.

Adicionalmente, a escolha da escala deve considerar a forma de construção e a dificuldade de manter a resposta do material o mais homogênea possível. Claramente, num caso hipotético, a geometria criada contorna esses problemas e estuda-se o método; para um caso real, a escolha de determinada escala permitirá soldas similares, que certamente aproximarão os resultados. Se a influência de algum fator não é conhecida, não sendo o objetivo estudá-la, ela deverá ser reproduzida na escala. Por fim, a resposta do modelo deve seguir a do protótipo, e não o contrário.

Apesar da dificuldade em se construir as geometrias em escala, os resultados dos ensaios em escala reduzida compensaram isso. Enquanto que a preparação do ensaio protótipo levou mais de 4 horas e 4 pessoas, a do modelo levou menos de 10 minutos e 2 pessoas, considerando posicionamento do painel, câmera e o ensaio propriamente dito. Além disso, há maior controle das variáveis do ensaio como direção do indentador, por causa das guias o manterem, como o posicionamento do painel; e até mesmo a periculosidade do ensaio, sendo bem menos ofensivo por causa da menor energia envolvida. Todos esses fatores indicam que a utilização de modelos e ensaios em escala economiza recursos, é mais seguro e propicia repetições mais facilmente.

\section{Mecanismo de Deformação}

O mecanismo de deformação assumido anteriormente aos ensaios e cujas equações foram desenvolvidas no Capítulo 5 foram os mesmos ocorridos nos paineis modelo após os ensaios, e o mesmo das análises numéricas, como pode ser visto ao confrontar as imagens do ensaio, Fig. 4.17, com as do modelo analítico, Figs. 5.4e e f e 5.5b; e as do numérico, Figs. 6.13 e 6.14. Isso mostra a qualidade da construção dos modelos em escala, bem 
como o controle do ensaio.

O mesmo não ocorreu para o painel protótipo, cuja geometria foi afetada pelo processo construtivo e o ensaio teve menor controle, resultando em um mecanismo de deformação que incluiu falha não prevista. Com esse ocorrido, a correlação entre os resultados experimentais com o uso das leis de similaridade desenvolvidas não foi boa, visto que os mecanismos não são similares. Contudo, analítica e numericamente isso pode ser feito, considerando os mecanismos que foram assumidos neste trabalho.

\section{Flexibilização de $\beta_{V}$ e $\beta_{G}$}

As restrições na preparação dos ensaios experimentais como velocidade e massa de impacto serviram de base para a ideia de flexibilizar os fatores $\beta_{V}$ e $\beta_{G}$, tornando alguns experimentos factíveis. Somente na conclusão do artigo sobre esse estudo (Mazzariol et al. (2011)), pôde ser observado que também seria possível enxergar os fatores sob outra ótica. Se o fator $\beta_{G}$ trata da massa do indentador e que, na verdade, tem a ver com toda e qualquer massa envolvida no experimento; então é possível pensar na massa da estrutura, ou melhor, o fator $\beta_{G}$ poderia compensar as densidades diferentes entre modelo e protótipo. Isto abre caminho para o desenvolvimento de leis de similaridade para diferentes materiais - de diferentes densidades, restando ainda questões sobre diferentes curvas tensão vs. deformação e diferentes respostas viscoplásticas.

\section{Fator de tensão de referência - $\beta_{\sigma_{0}}$}

Embora escolhidos materiais nominalmente próximos, suas curvas tensão-deformação não se mostraram similares. Tampouco essa condição fora anteriormente considerada ou enfrentada de maneira tão crítica como neste trabalho. A escolha de $\beta_{\sigma_{0}}$ em função de $\sigma_{Y}$, mostrou-se fundamentada quando se considera o volume deformado esperado, i.e., a deformação média que ocorrerá na estrutura impactada. Se hipoteticamente considerarmos para o cáculo de $\beta_{\sigma_{0}}$ toda a curva $\sigma \times \varepsilon$ e houver pouca deformação, estaremos superestimando a resposta, que será inferior à do protótipo e, portanto, não correlacionará apropriadamente o evento.

Um exemplo numérico simplificado de uma chapa retangular de dimensões $200 \mathrm{x}$ 800 x $3 \mathrm{~mm}$ engastada nas extremidades e impactada por uma massa de $957 \mathrm{~kg}$ a $5 \mathrm{~m} / \mathrm{s}$ no centro, tal que sofresse deformação da ordem de $50 \%$, foi resolvido por elementos finitos com alguns resultados na Fig. 7.2. Os resultados obtidos com escala $\beta=1 / 16$, e 
usando $\beta_{\sigma_{0}}=\sigma_{Y} / \sigma_{Y}$ corroboram os comentários acima. Se usássemos a curva tensão vs. deformação inteira, $\left(\beta_{\sigma_{0}}=0,87\right.$, Tabela 3.4$)$, curva "verde", teríamos um resultado mais aproximado, inclusive mais próximo do protótipo do que o MLT. Dessa forma, o método de estimar a deformação média através da transferência de energia pode auxiliar nesses casos de curvas bastante distintas, ainda que seja sempre desejável que não seja preciso conhecer a resposta da estrutura para poder escaloná-la.

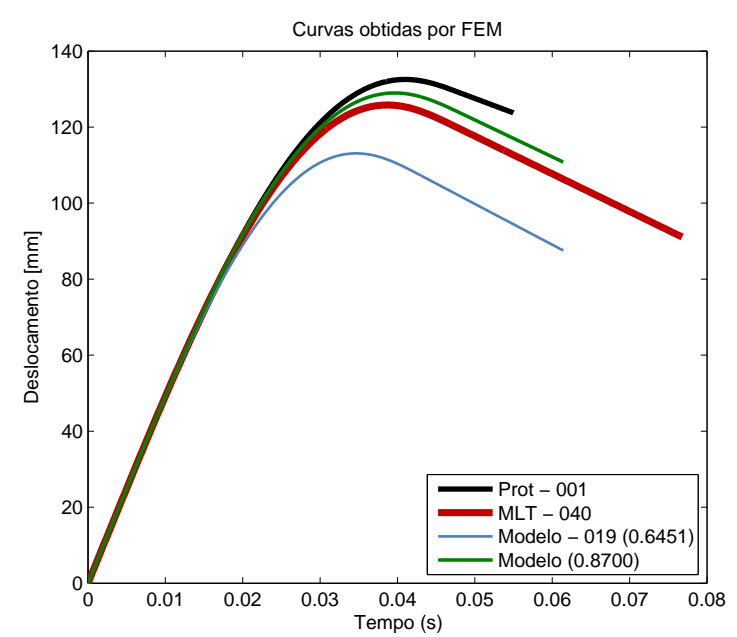

(a) Deslocamento corrigido vs. tempo corrigido.

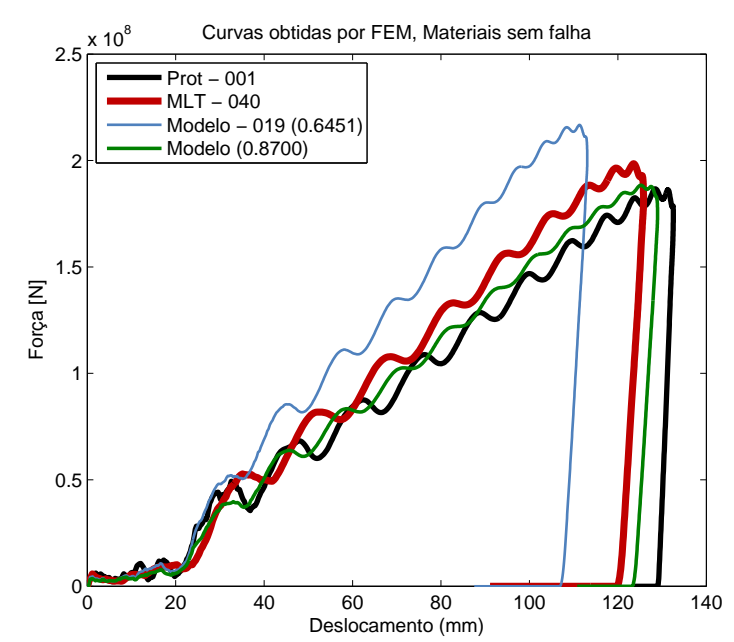

(b) Força corrigida vs. deslocamento corrigido.

Figura 7.1 Correlação numérica entre protótipo, modelo MLT e duas abordagens VSG com diferentes fatores $\beta_{\sigma_{0}}$.

\section{Caracterização do Material}

A caracterização dos materiais realizada no Capítulo 3 compreendeu ensaios a baixas e altas taxas de deformação, não sendo possível realizar ensaios à médias taxas por limitação dos equipamentos existentes. Uma caracterização que compreendesse essa faixa intermediária de taxa de deformação permitiria que o comportamento viscoplástico fosse mais bem captado, provavelmente reduzindo as discrepâncias numérico-experimentais das Figs. 6.10 e 6.11. Além disso, sendo as curvas quase-estáticas para as duas espessuras de material diferentes, existe a possibilidade de o comportamento dinâmico do material também o ser, como as curvas de regressão linear do material das Figs. 3.25 e 3.26 levam a concluir. Enfim, a escolha do material para o modelo necessita de uma compreensão bastante ampla da resposta do material para o protótipo, tanto estática como dinâmica. 


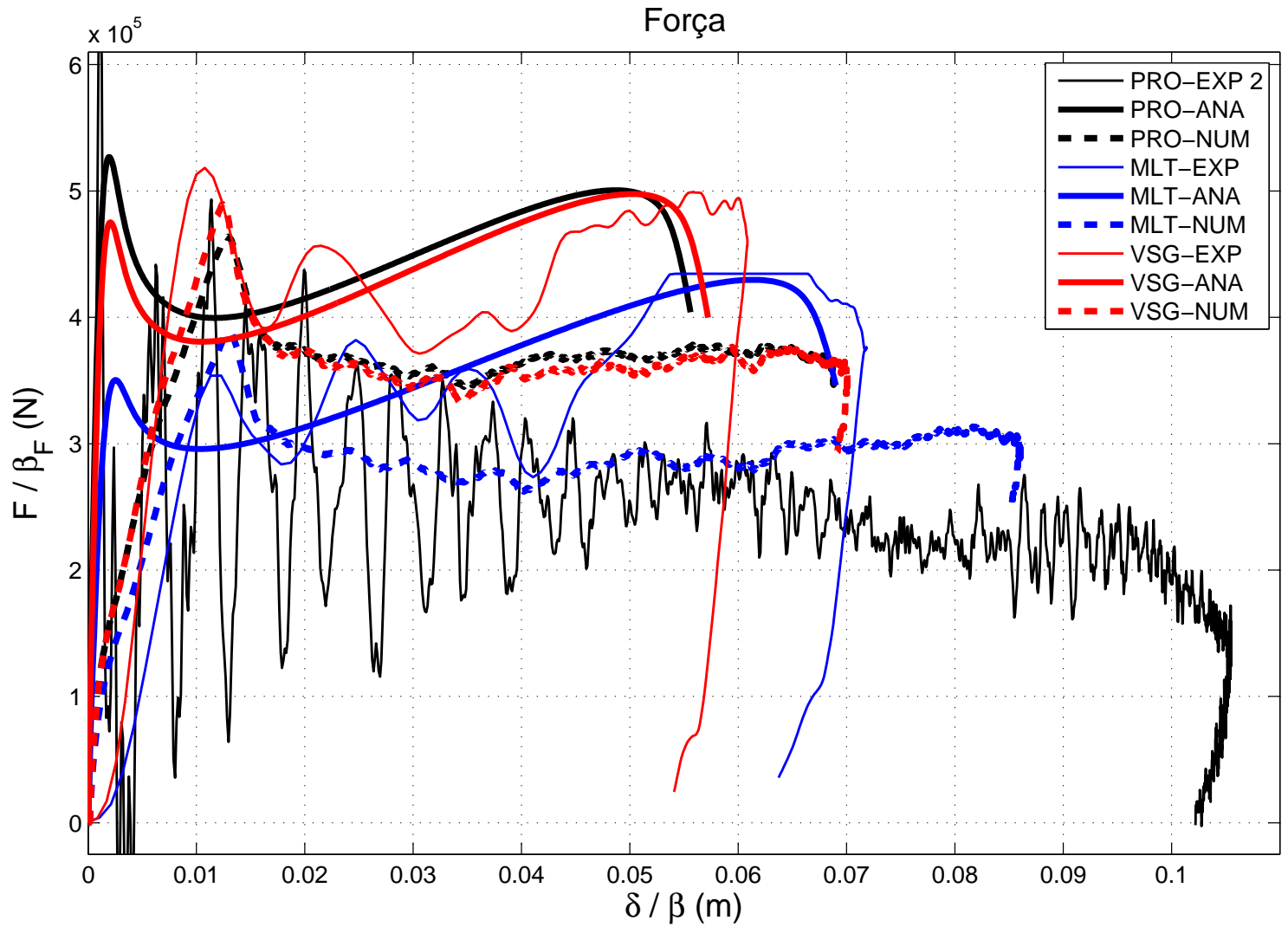

(a) Força corrigida vs. deslocamento corrigido.

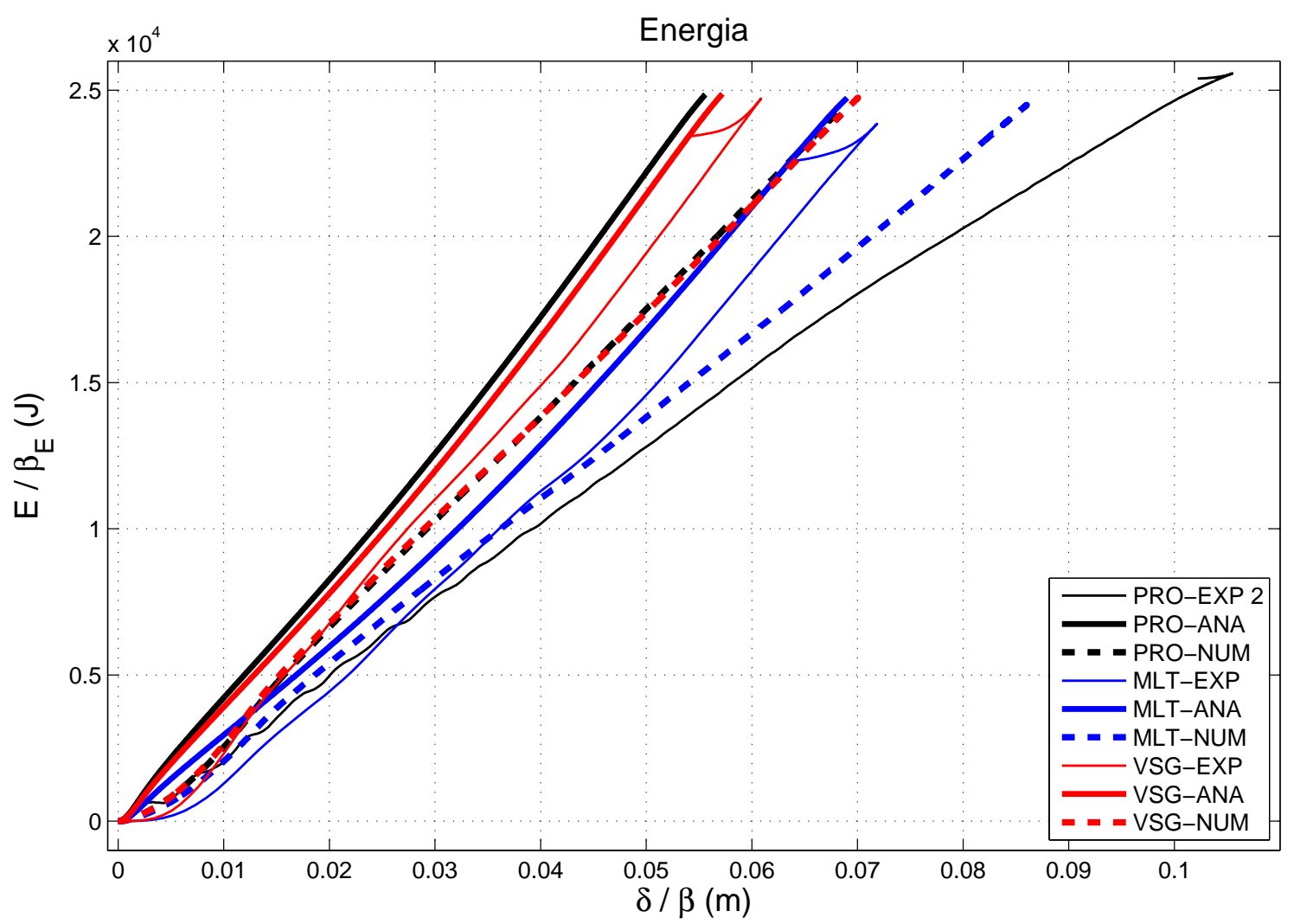

(b) Energia corrigida vs. deslocamento corrigido.

Figura 7.2 Curvas corrigidas para protótipo 2 e modelos MLT e VSG para as abordagens analítica, numérica e experintal. 
- Comentários Finais

\section{Resultados}

Nas Figs.7.2a e 7.2b encontram-se os resultados experimental, analítico e numéricos obtidos para os paineis protótipos e modelos MLT e VSG nos Capítulos 4, 5 e 6; juntando a comparação entre resultados experimentais das Figs. 4.18b e 4.19b; analíticos das Figs. 5.19a e 5.19b; e numéricos das Figs. 6.12a e 6.12b. 


\section{CAPÍtulo 8}

\section{Conclusões}

Neste trabalho foi apresentada uma técnica para efetuar o escalonamento de uma estrutura de painel reforçado sujeita ao impacto de um indentador. A conhecida resposta "mais rígida" em escala foi contornada com o uso de leis de similaridade distorcidas, ao modificar a massa e a velocidade inicial do elemento impactante. As diferenças no material de modelo e protótipo foram levadas em consideração e discutidas para o presente estudo, sendo uma aplicação de conceito estendida das leis originalmente propostas por Oshiro (2010). A essas equações de correlação foi adicionado o conceito de flexibilização da velocidade inicial, para contornar limitações experimentais.

Para avaliar a técnica proposta foram ensaiados dois paineis protótipos como referência, um painel em escala utilizando o método convencional de similaridade, com a base Massa-Comprimento-Tempo (MLT) e um último painel em escala que utilizou as leis distorcidas, na base Velocidade-Tensão de Escoamento - Massa de Impacto, ou VSG. De forma a confrontar esses resultados, o comportamento desses paineis foram também descritos analiticamente e avaliados através de simulação numérica.

Os resultados experimentais do painel protótipo não tiveram boa correlação com os numéricos e os analíticos devido à fratura dos paineis. Contudo, o resultado experimental de ambos os paineis em escala foram bem descritos pelas equações analíticas e pelas simulações. Com a aplicação das leis de similaridade distorcidas, técnica apresentada neste trabalho, foi possível obter respostas dos modelos VSG bastante próximas do protótipo, tanto para o modelo analítico quanto para as simulações.

Por fim, os resultados mostram a viabilidade de se contornar limitações experimentais em um ensaio de protótipo usando modelos e, eventualmente, contornar características 
dessa representação - como diferentes materiais, com a avaliação caso a caso das curvas tensão vs. deformação. A técnica aprimorada no presente trabalho abre caminhos para a utilização de materiais de densidade e comportamento geral distintos, flexibilização das condições de ensaio para mesma escala etc., estendendo a aplicação proposta por Oshiro (2010). 


\section{Referências}

ALSOS, H. S.; AMDAHL, J. On the resistance of tanker bottom structures during stranding. Marine Structures, v. 20, n. 4, p. 218 - 237, 2007. ISSN 0951-8339. Disponível em: $<$ http://www.sciencedirect.com/science/article/pii/S0951833907000342>. . On the resistance to penetration of stiffened plates, part i - experiments. International Journal of Impact Engineering, v. 36, n. 6, p. 799 - 807, 2009. ISSN 0734-743X. Disponível em: $<$ http://www.sciencedirect.com/science/article/pii/S0734743X08002753>.

ALSOS, H. S.; AMDAHL, J.; HOPPERSTAD, O. S. On the resistance to penetration of stiffened plates, part ii: Numerical analysis. International Journal of Impact Engineering, v. 36, n. 7, p. 875 - 887, 2009. ISSN 0734-743X. Disponível em: $<$ http://www.sciencedirect.com/science/article/pii/S0734743X08003072>.

AMDAHL, J. Energy Absorption in Ship-Platform Impact. Trondheim, Noruega, 1983.

BAKER, W. E.; WESTINE, P. S.; DODGE, F. T. Similarity Methods in Engineering Dynamics: Theory and Practice of Scale Modeling. 1st. ed. Amsterdam: Elsevier Science Publishers, 1991. $348 \mathrm{p}$.

BARENBLATT, G. Scaling. [S.l.]: Cambridge University Press, 2003. (Cambridge Texts in Applied Mathematics). ISBN 978-0-521-53394-2.

BATHE, K. Finite element procedures. [S.1.]: Prentice Hall, 1996. 475-476;768-829 p. ISBN 9783642090028 .

BENDICK, J.; GLEISER, M. Arquimedes - uma porta para a ciência. [S.l.]: Odysseus, 2006. (Imortais da ciência). ISBN 9788588023789. 
BOOTH, E.; COLLIER, D.; MILES, J. Impact scalability of plated steel structures. In: JONES, N.; WIERZBICKI, T. (Ed.). Structural Crashworthiness. London: Butterworths \& Co. Publishers, 1983. p. $136-174$.

CARLEBUR, A. Full-scale collision tests. Safety Science, v. 19, n. 2-3, p. $171-178$, 1995. ISSN 0925-7535. <ce:title $>$ Safety of Transportation $</$ ce:title $>$. Disponível em: <http://www.sciencedirect.com/science/article/pii/092575359400018X>.

CHO, S.-R.; LEE, H.-S. Experimental and analytical investigations on the response of stiffened plates subjected to lateral collisions. Marine Structures, v. 22, n. 1, p. 84 - 95, 2009. ISSN 0951-8339. < ce:title>Selected papers from the 3rd International Conference on Collision and Grounding of Ships $</$ ce:title $>$. Disponível em: $<$ http://www.sciencedirect.com/science/article/pii/S0951833908000270>.

DAWSEY, J. C. Por uma antropologia benjaminiana: repensando paradigmas do teatro dramático. Mana, scielo, v. 15, p. 349 - 376, 10 2009. ISSN 0104-9313. Disponível em: $<$ http://www.scielo.br/scielo.php?script=sci_arttext\&pid=S0104-93132009000200002\&nrm $=$ iso $>$.

ENDO, H.; YAMADA, Y.; KITAMURA, O.; SUZUKI, K. Model test on the collapse strength of the buffer bow structures. Marine Structures, v. 15, n. 4-5, p. $365-381$, 2002. ISSN 0951-8339. < ce:title>Ship Collisions and Grounding</ce:title>. Disponível em: $<$ http://www.sciencedirect.com/science/article/pii/S0951833902000084>.

FOX, R. W.; MCDONALD, A. T. Introduction to Fluid Mechanics. 4th. ed. New York: John Wiley \& Sons, 1998. 192-206 p.

HUTCHINSON, B. L. Barge collisions, ramming and groundings - an engineering assessment of the potential for damage to radioactive material transport casks. [S.l.], 1986.

JONES, N. Structural Impact. 1st. ed. New York, USA: Cambridge University Press, 1997. 575 p.

KITAMURA, O. Fem approach to the simulation of collision and groun-

ding damage. Marine Structures, v. 15, n. 4-5, p. 403 - 428, 2002. ISSN

0951-8339. < ce:title $>$ Ship Collisions and Grounding $</$ ce:title $>$. Disponível em: <http://www.sciencedirect.com/science/article/pii/S0951833902000102>.

LEHMANN, E.; PESCHMANN, J. Energy absorption by the steel structure of ships in the event of collisions. Marine Structures, v. 15, n. 4-5, p. $429-441,2002$. ISSN 0951-8339. < ce:title >Ship Collisions and Grounding </ce:title $>$. Disponível em: <http://www.sciencedirect.com/science/article/pii/S0951833902000114>.

LEMAITRE, J.; CHABOCHE, J. L. Mechanics of solids materials. 1st. ed. Cambridge: Cambridge University Press, 1991. 584p p. 
LIVERMORE SOFTWARE TECHNOLOGY CORPORATION. LS-DYNA Theory Manual.Livermore Software Technology Corporation. Livermore, California,EUA, 2006. $680 \mathrm{p}$.

LS-DYNA Keyword User's Manual. Livermore Software Technology Corporation. version 971, revision 1. Livermore, California,EUA, 2007. 2206 p.

MAZZARIOL, L. M.; OSHIRO, R. E.; CALLE, M. A. G.; ALVES, M. Scaling of stiffened panels subjected to impact load. In: DVORKIN, E. N.; GOLDSCHMIDT, M. B. (Ed.). MECOM-CILAMCE. Buenos Aires: AMCA, 2010. p. 1275-1289.

MAZZARIOL, L. M.; OSHIRO, R. E.; CALLE, M. A. G.; MOURA, R. T.; ALVES, M. Evaluating velocity and mass correction on scaling of structures subjected to impact loading. In: FANCELLO, E.; TARÇO, P. de; MENDONÇA, R.; ALVES, M. (Ed.). Proceedings of the International Symposium on Solid Mechanics. Florianopolis. Florianopolis, SC, Brazil: ABCM, 2011. p. 353-366.

MINORSKY, V. U. Analysis of ship collision with reference to protection of nuclear power ships. Journal of Ship Reseach, v. 3, n. 2, p. 1 - 4, 1959.

MOURA, R. T. Correção da dispersão de sinais na caracterização dinâmica de materiais utilizando a barra de Hopkinson. Trabalho de Formatura - Escola Politécnica da Universidade de São Paulo, São Paulo, Brasil, 2006.

MURPHY, G. Similitude in engineering. [S.l.]: Ronald Press Co., 1950.

OHTSUBO, H.; KAWAMOTO, Y.; KUROIWA, T. Experimental and numerical research on ship collision and grounding of oil tankers. Nuclear Engineering and Design, v. 150, n. 2-3, p. $385-396,1994$. ISSN 0029-5493. Disponível em: <http://www.sciencedirect.com/science/article/pii/0029549394901589>.

OSHIRO, R.; ALVES, M. Scaling of structures subject to impact loads when using a power law constitutive equation. International Journal of Solids and Structures, v. 46, n. 18-19, p. 3412-3421, 2009. ISSN 0020-7683. Disponível em: $<$ http://www.sciencedirect.com/science/article/pii/S0020768309002212>.

OSHIRO, R. E. Estudo da similaridade imperfeita em estruturas sujeitas a carregamentos de impacto. Tese (Doutorado em Engenharia Mecânica) - EPUSP, Sao Paulo, 2010. In Portuguese. OSHIRO, R. E.; CALlE, M. A. G.; MAZZARIOL, L. M.; ALVES, M. Experimental study of scaled $\mathrm{t}$ cross-section beams subjected to impact load. In: Proceedings of 21st Brazilian Congress of Mechanical Engineering. Natal, RN, Brasil: ABCM, 2011. p. 10p. ISSN 2176-5480.

OZGUC, O.; DAS, P. K.; BARLTROP, N. A comparative study on the structural integrity of single and double side skin bulk carriers under collision damage. Marine Structures, v. 18, n. 7-8, p. 511 - 547, 2005. ISSN 0951-8339. Disponível em: $<$ http://www.sciencedirect.com/science/article/pii/S0951833906000153>. 
PAIK, J.; THAYAMBALLI, A. Ultimate Limit State Design of Steel-Plated Structures. [S.l.]: J. Wiley, 2003. ISBN 9780471486329.

PAIK, J. K.; AMDAHL, J.; BARLTROP, N.; DONNER, E. R.; GU, Y. (Ed.). Committee V.3 Collision and Grounding of International Ship and Offshore Structures Congress. San Diego, USA, August 2003. 37 p.

PEDERSEN, P. T.; VALSGåRD, S.; OLSEN, D.; SPANGENBERG, S. Ship impacts: Bow collisions. International Journal of Impact Engineering, v. 13, n. 2, p. 163 - 187, 1993. ISSN 0734-743X. Disponível em: <http://www.sciencedirect.com/science/article/pii/0734743X9390091K>.

PETERSEN, M. J. Dynamics of ship collisions. Ocean Engineering, v. 9, n. 4, p. 295 - 329, 1982. ISSN 0029-8018. Disponível em: <http://www.sciencedirect.com/science/article/pii/0029801882900269>. PETROBRÁS TRANSPORTE S.A. Programa de Navios: Descrição breve do programa de modernização e expansão da frota. 2012. Disponível em: <http://www.transpetro.com.br, Acesso em "Atividades", "Transporte Marítimo", "Programa de Navios">.

RAMESH, K. T. High rates and impact experiments. In: SHARPE, W. N. (Ed.). Springer Handbook of Experimental Solid Mechanics. Springer US, 2008. p. 929-960. ISBN 978-0-387-30877-7. 10.1007/978-0-387-30877-7-33. Disponível em: <http://dx.doi.org/10.1007/978-0-387-30877-7-33>. SERVIS, D. P.; SAMUELIDES, M. Ship collision analysis using finite elements. In: Proceeding of SAFER EURORO SPRING MEETING, NANTES. Athens, Greece: [s.n.], 1999.

SINDICATO NACIONAL DA INDúSTRIA DA CONSTRUçãO E REPARAçãO NAVAL E OFFSHORE - SINAVAL. Balanço 2008: Principais acontecimentos, construções em andamento, capacidade produtiva e tecnologia naval. Rio de Janeiro: Sindicato Nacional da Indústria da Construção e Reparação Naval e Offshore, 2008.

SKOGLUND, V. J. Similitude - Theory and Applications. [S.l.]: International Textbook Company, 1967. $320 \mathrm{p}$.

SOARES, C. G.; BASU, R.; SIMONSEN, B. C.; EGOROV, G. V.; HUNG, C. F. (Ed.), 17., 2009, Seoul, Korea. Committee V.1 Damage Assessment After Accidental Events of International Ship and Offshore Structures Congress. Seoul, Korea: [s.n.], 2009. 72 p.

SUTTON, M. A. Digital image correlation for shape and deformation measurements. In: SHARPE, W. N. (Ed.). Springer Handbook of Experimental Solid Mechanics. Springer US, 2008. p. 565-600. ISBN 978-0-387-30877-7. 10.1007/978-0-387-30877-7-20. Disponível em: $<$ http://dx.doi.org/10.1007/978-0-387-30877-7-20>.

TABRI, K.; MÄÄTTÄNEN, J.; RANTA, J. Model-scale experiments of symmetric ship collisions. Journal of Marine Science and Technology, Springer Japan, v. 13, p. 71-84, 2008. ISSN 0948-4280. 10.1007/s00773-007-0251-z. Disponível em: <http://dx.doi.org/10.1007/s00773-007-0251-z>. 